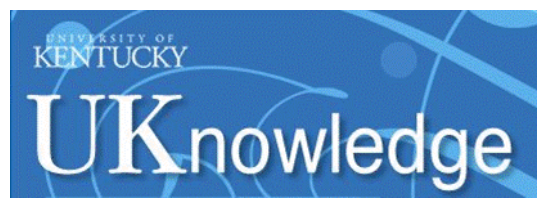

University of Kentucky

UKnowledge

\title{
Properties of Dust Grains in Planetary Nebulae. I. The lonized Region of NGC 6445
}

\author{
Peter A. M. van Hoof \\ University of Kentucky \\ Griet C. Van de Steene \\ Mount Stromlo Observatory, Australia \\ Douwe A. Beintema \\ Laboratory for Space Research, The Netherlands \\ P. G. Martin \\ University of Toronto, Canada \\ Stuart R. Pottasch \\ Kapteyn Astronomical Institute, The Netherlands
}

See next page for additional authors

Follow this and additional works at: https://uknowledge.uky.edu/physastron_facpub

Part of the Astrophysics and Astronomy Commons, and the Physics Commons

Right click to open a feedback form in a new tab to let us know how this document benefits you.

\section{Repository Citation}

van Hoof, Peter A. M.; Van de Steene, Griet C.; Beintema, Douwe A.; Martin, P. G.; Pottasch, Stuart R.; and Ferland, Gary J., "Properties of Dust Grains in Planetary Nebulae. I. The lonized Region of NGC 6445" (2000). Physics and Astronomy Faculty Publications. 111.

https://uknowledge.uky.edu/physastron_facpub/111

This Article is brought to you for free and open access by the Physics and Astronomy at UKnowledge. It has been accepted for inclusion in Physics and Astronomy Faculty Publications by an authorized administrator of UKnowledge. For more information, please contact UKnowledge@lsv.uky.edu. 
Properties of Dust Grains in Planetary Nebulae. I. The lonized Region of NGC 6445

Digital Object Identifier (DOI)

http://dx.doi.org/10.1086/308536

Notes/Citation Information

Published in The Astrophysical Journal, v. 532, no. 1, p. 384-399.

(c) 2000. The American Astronomical Society. All rights reserved. Printed in the U.S.A.

The copyright holder has granted permission for posting the article here.

Authors

Peter A. M. van Hoof, Griet C. Van de Steene, Douwe A. Beintema, P. G. Martin, Stuart R. Pottasch, and Gary J. Ferland 
THE ASTROPHYSICAL JOURNAL, 532:384-399, 2000 March 20

(C) 2000. The American Astronomical Society. All rights reserved. Printed in U.S.A.

\title{
PROPERTIES OF DUST GRAINS IN PLANETARY NEBULAE. I. THE IONIZED REGION OF NGC 6445
}

\author{
Peter A. M. VAN HoOF ${ }^{1}$ \\ University of Kentucky, Department of Physics and Astronomy, 177 CP Building, Lexington, KY 40506-0055; peter@pa.uky.edu
}

Griet C. VAN De STEENE

Research School of Astronomy and Astrophysics, Mount Stromlo Observatory, Private Bag, Weston Creek, ACT 2611, Australia; gsteene@mso.anu.edu.au

DOUWE A. BeINTEMA

Laboratory for Space Research, Postbus 800, NL-9700 AV Groningen, The Netherlands; douwe@sron.rug.nl

\section{P. G. Martin}

Canadian Institute for Theoretical Astrophysics, McLennan Laboratories, University of Toronto, 60 St. George Street, Toronto, ON M5S 3H8, Canada; pgmartin@cita.utoronto.ca

STUART R. PotTasch

Kapteyn Astronomical Institute, Postbus 800, NL-9700 AV Groningen, The Netherlands; pottasch@astro.rug.nl

\author{
AND
}

GARY J. FERLAND ${ }^{1}$

University of Kentucky, Department of Physics and Astronomy, 177 CP Building, Lexington, KY 40506-0055; gary@cloud9.pa.uky.edu Received 1999 February 17; accepted 1999 November 11

\begin{abstract}
One of the factors influencing the spectral evolution of a planetary nebula is the fate of the dust grains that are emitting the infrared continuum. Several processes have been proposed that either destroy the grains or remove them from the ionized region. To test whether these processes are effective, we study new infrared spectra of the evolved nebula NGC 6445. These data show that the thermal emission from the grains is very cool and has a low flux compared to $\mathrm{H} \beta$. A model of the ionized region is constructed, using the photoionization code CLOUDY 90.05. Based on this model, we show from depletions in the gas-phase elements that little grain destruction can have occurred in the ionized region of NGC 6445. We also argue that dust-gas separation in the nebula is not plausible. The most likely conclusion is that grains are residing inside the ionized region of NGC 6445 and that the low temperature and flux of the grain emission are caused by the low luminosity of the central star and the low optical depth of the grains. This implies that the bulk of the silicon-bearing grains in this nebula were able to survive exposure to hard-UV photons for at least several thousands of years, contradicting previously published results. A comparison between optical and infrared diagnostic line ratios gives a marginal indication for the presence of a $t^{2}$ effect in the nebula. However, the evidence is not convincing and the differences could also be explained by uncertainties in the absolute flux calibration of the spectra, the aperture corrections that have been applied, or the collisional cross sections. The photoionization model allows an accurate determination of the central star temperature based on model atmospheres. The resulting value of $184 \mathrm{kK}$ is in good agreement with the average of all published Zanstra temperatures based on blackbody approximations. The off-source spectrum taken with LWS clearly shows the presence of a warm cirrus component with a temperature of $24 \mathrm{~K}$ as well as a very cold component with a temperature of 7 $\mathrm{K}$. Since our observation encompasses only a small region of the sky, it is not clear how extended the 7 $\mathrm{K}$ component is and whether it contributed significantly to the Far-Infrared Absolute Spectrophotometer (FIRAS) spectrum taken by COBE. Because our line of sight is in the Galactic plane, the very cold component could be a starless core.
\end{abstract}

Subject headings: circumstellar matter - dust, extinction - infrared: ISM: continuum planetary nebulae: individual (NGC 6445) - stars: AGB and post-AGB stars: fundamental parameters

\section{INTRODUCTION}

When a low- or intermediate-mass star is approaching the end of its evolution, it goes through a period of heavy mass loss known as the asymptotic giant branch (AGB) stage. In this phase the star is a red giant, which consists of a degenerate $\mathrm{C} / \mathrm{O}$ core surrounded by a very tenuous envelope. During the AGB stage the star loses a substantial fraction of its initial mass, eventually leaving only a degenerate core of typically $0.6-0.8 M_{\odot}$ covered with a thin layer

\footnotetext{
${ }^{1}$ Visiting Astronomer at the Canadian Institute for Theoretical Astrophysics, Toronto, Canada.
}

of hydrogen. This core will eventually evolve into a white dwarf. It is generally assumed that dust formation is very efficient in the AGB outflow, so large amounts of dust grains are formed during that phase. Once the envelope mass of the central star drops below a critical value, the mass-loss rate will drop several orders of magnitude and the star will enter the post-AGB phase of its evolution. During this phase the central star will heat up and the AGB ejecta will expand, forming a detached shell. Once the central star reaches a temperature of $20-30 \mathrm{kK}$, it will start to ionize the AGB shell and a planetary nebula (PN) will form. After this the central star will continue heating up at roughly constant luminosity until finally the nuclear reactions cease and the 


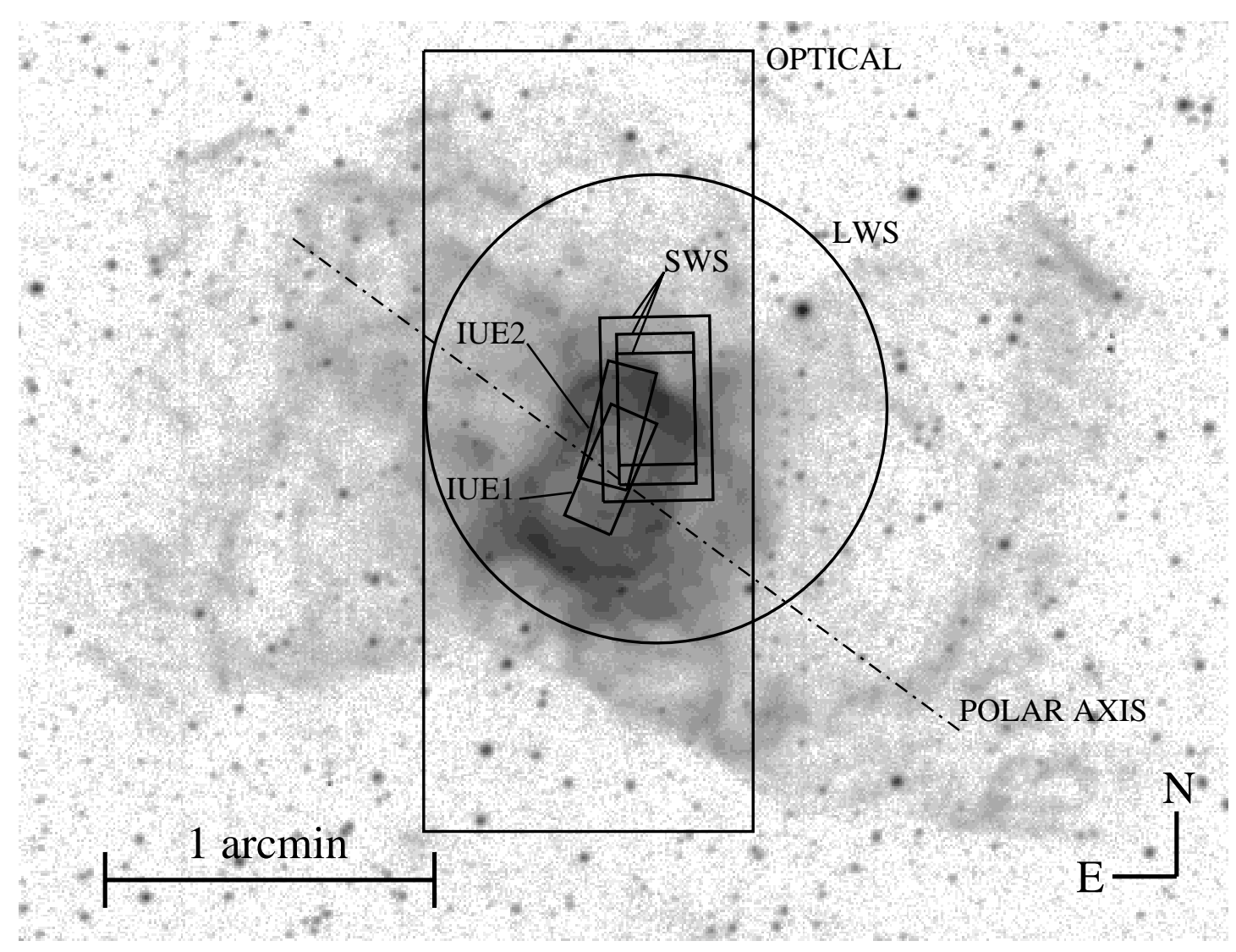

FIG. 1.- Various apertures overplotted on an $\mathrm{H} \alpha+[\mathrm{N} \mathrm{II}]$ image of NGC 6445 (Schwarz et al. 1992). The intensity has been scaled logarithmically to emphasize the low surface brightness regions.

central star decreases both in temperature and luminosity. All this time the detached AGB shell will continue to expand and undergo hydrodynamic interactions with the fast wind emanating from the central star. When the central star enters the cooling track, the number of ionizing photons emitted by the star will decline sharply. If this decline in luminosity is fast enough (i.e., if the central star is massive enough), this will cause the ionized region to shrink, and the outermost parts of the nebula will start recombining. Eventually the cooling rate of the central star will slow down and the nebula will dissolve in the interstellar medium (ISM), leaving a bare white dwarf. Both the evolution of the central star and the expansion of the AGB ejecta will have a profound effect on the infrared emission

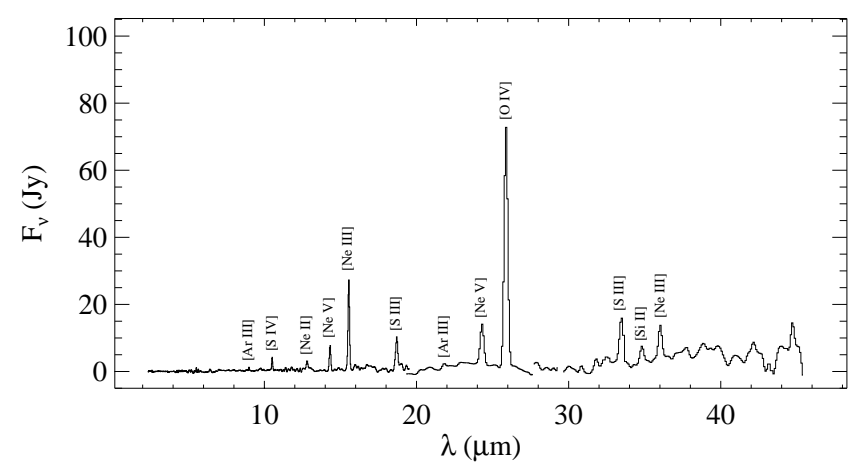

FIG. 2.-ISO-SWS spectrum of the planetary nebula NGC 6445 from the dust grains, causing a substantial evolution of both the total infrared flux and the temperature of the dust grains.

During the post-AGB phase, the combined effects of increasing stellar temperature (which will increase the grain temperature) and nebular expansion (which will decrease the grain temperature) cannot be predicted easily, and detailed modeling is necessary. A comprehensive discussion of the evolution of the infrared spectrum during this phase can be found in van Hoof, Oudmaijer, \& Waters (1997a). However, after the central star has reached a temperature of $40-50 \mathrm{kK}$, the effect of the rising stellar temperature on the grains will be reversed. This is caused by the fact that the peak of the stellar spectrum shifts to ever shorter wavelengths, beyond the peak of the grain absorption cross section, thereby making grain heating less efficient. Therefore, once the nebula is in the PN phase, the evolution of the infrared spectrum is clear. Both the ratio of the total infrared flux over the $\mathrm{H} \beta$ flux and the average dust temperature will decrease with increasing (Strömgren) radius of the nebula. However, a considerable amount of scatter around this average evolution can be expected in a statistical sample because of the particulars of the evolution of each individual nebula.

One of the factors influencing the evolution of the infrared spectrum is the dust grains themselves. In the previous discussion we implicitly assumed that nothing much happened to the grains; however, this is not at all clear. It has been proposed that the dust grains could be destroyed as the PN evolves (e.g., Natta \& Panagia 1981; Pottasch 1987), 
either because of spallation by hard UV photons or because of shocks. It is known that UV photons are abundantly present in $\mathrm{PNe}$, as is the case for shocks that are formed because of the interaction of the fast wind with the slow AGB ejecta. Hence, if these processes are efficient, the ionized regions of evolved PNe should be free of dust grains. Another possibility is that dust grains are separated from the gas because of radiation pressure. This could lead to a general flow of the grains with respect to the gas or, if instabilities occur, to the formation of small, high-density globules that would be neutral or partially ionized. Such globules have been observed, suggesting that at least in some nebulae this mechanism is effective.

To investigate these points in more detail, we shall analyze the infrared spectrum of an evolved PN and search for indications of any of the processes we mentioned above. This nebula is NGC 6445, and it is classified as a bipolar type I PN (Corradi \& Schwarz 1995; Perinotto 1991, respectively), a class assumed to originate from massive progenitors. Based on its low density, the nebula appears well evolved. Both the ionized and neutral material (CO) show high expansion velocities, $38 \mathrm{~km} \mathrm{~s}^{-1}$ (Weinberger 1989) and $33 \mathrm{~km} \mathrm{~s}^{-1}$ (Huggins \& Healy 1989), respectively. These properties are consistent with a central star that was luminous in the early stages of its post-AGB evolution and therefore of high mass. Also, the high central star temperature we find (see $\S 8$ ) is consistent with this. An image of the nebula is shown in Figure 1. We have obtained an SWS spectrum of this nebula, which is remarkable in the fact that it shows virtually no dust emission (see Fig. 2). This is indicative of a low $F(\mathrm{IR}) / F(\mathrm{H} \beta)$ ratio and dust temperature for this object, which confirms the evolved status of this PN. It also makes the object very suitable to investigate the points mentioned earlier.

First we will discuss the observations gathered for this source in $\S 2$. Since the nebula is very large, aperture corrections are needed. These are discussed in $\S 3$. Next we discuss the reddening correction of the spectra in $\S 4$. Some details concerning the observed spectrum, including the possible influence of a recombining halo, are discussed in $\S 5$. Nebular conditions derived from plasma diagnostics and the possible presence of $t^{2}$ effects are discussed in $\S 6$. Then we study the observed thermal grain emission in $\S 7$. Finally we construct a photoionization model of the nebula in $\S 8$, which is used to study the possible presence of dust grains inside the ionized region of the nebula. Final conclusions are given in $\S 9$.

\section{OBSERVATIONS}

\subsection{Unpublished Data}

In the analysis of NGC 6445 several spectra were used, covering a wide range of wavelengths. A log of all the observations that were not previously published can be found in Table 1. The International Ultraviolet Explorer (IUE) spectra were taken from the IUE Newly Extracted Spectra (INES) catalog in the IUE final archive. No further processing was done on these data. For further reference the SWP17030 spectrum will be called IUE1 and the SWP38267 spectrum IUE2. The LWP17434 spectrum does not contain any detected lines and will not be discussed further.

The optical observations were done with the Boller \& Chivens spectrograph at the $1.52 \mathrm{~m}$ ESO telescope on La Silla. CCD 24, which is a Ford Aerospace 2048L, and grating 25 were used. At a grating angle of $8^{\circ}$ this gave us wavelength coverage from 391.4 to $967.8 \mathrm{~nm}$, or $0.283 \mathrm{~nm}$ pixel $^{-1}$ and a resolution of about $0.7 \mathrm{~nm}$. We were not affected by the second-order spectrum with this setting. A special technique was used to obtain large-aperture exposures. For this the slit of the spectrograph was oriented north-south and the tracking rate of the telescope in the east-west direction was offset by $0.05 \mathrm{~s}^{-1}$. This caused the slit to gradually drift over the nebula, causing the spectrograph to integrate photons from the entire nebula. The exposure time listed in Table 1 is the effective exposure time, i.e., the slit width divided by the differential tracking rate times the number of exposures. This technique allows the optical spectrum to be directly comparable to other largeaperture data.

The reduction was done using standard procedures in IRAF. First the spectra were bias subtracted and flat fielded with a normalized dome flat. The spectra were corrected for slit illumination. The images were geometrically corrected to rectify the spatial axis before sky subtraction. The dispersion axis is parallel to the chip's axis well within 2 pixels along the slit. This was good enough for our purposes as the spatial extent for the untraceable $\mathrm{PN}$ was much larger than

TABLE 1

LOG OF OBSERVATIONS

\begin{tabular}{|c|c|c|c|c|c|c|c|c|}
\hline \multirow[b]{2}{*}{ TELESCOPE } & \multirow[b]{2}{*}{ INSTRUMENT } & \multirow[b]{2}{*}{ DATE } & \multirow{2}{*}{$\begin{array}{c}\text { EXPOSURE } \\
\text { (s) }\end{array}$} & \multirow{2}{*}{$\begin{array}{l}\text { APERTURE }^{\mathrm{a}} \\
(\operatorname{arcsec})\end{array}$} & \multirow{2}{*}{$\begin{array}{l}\text { P.A. } \\
\text { (deg) }\end{array}$} & \multicolumn{2}{|c|}{ Central Position (2000.0) } & \multirow{2}{*}{$\begin{array}{l}\text { SPECTRAL RANGE } \\
(\mu \mathrm{m})\end{array}$} \\
\hline & & & & & & $\alpha$ & $\delta$ & \\
\hline \multirow{2}{*}{$I U E \ldots \ldots \ldots \ldots \ldots$} & SWP38267 & 900228 & 3600 & $9.07 \times 21.65$ & 165.5 & 174914.9 & -200026 & $0.115-0.198$ \\
\hline & LWP17434 & 900227 & 3600 & $9.91 \times 22.51$ & 165.5 & 174914.9 & -200026 & $0.185-0.336$ \\
\hline ESO $1.52 \mathrm{~m} \ldots \ldots$ & $\mathrm{B} \& \mathrm{Ch}$ & 950516 & $120^{\mathrm{c}}$ & $60 \times 140$ & 0.0 & 174914.8 & -200040 & $0.391-0.968$ \\
\hline \multirow{2}{*}{ ISO .............. } & LWS01 & 970317 & $640^{\mathrm{d}}$ & $\varnothing 84$ circular & $\ldots$ & 174914.4 & -200024 & $43.2-189$ \\
\hline & LWS01 OFF & 970317 & $640^{\mathrm{d}}$ & $\varnothing 84$ circular & $\ldots$ & 174915.3 & -195734 & $43.2-189$ \\
\hline
\end{tabular}

${ }^{\text {a }}$ The dimensions are EW $\times$ NS, assuming P.A. $=0^{\circ}$.

b The aperture rotates counterclockwise for increasing position angle.

c This is the effective exposure time (see $\S 2$ ).

d This is the target dedicated time.

e The aperture size depends on the wavelength (see $\S 3$ ).

${ }^{\mathrm{f}}$ Selected emission lines were observed (see Table 3 ). 
this. The standard stars were traced when extracted. The PN spectra were extracted with a fixed aperture based on the emission line with the largest spatial extent. The spectra were corrected for airmass and sensitivity with the response curve based on the standard star LTT 7379. Finally the three spectra were averaged with min-max rejection.

The infrared data were all taken with the Infrared Space Observatory (ISO), using the Short Wavelength Spectrometer (SWS) and the Long Wavelength Spectrometer (LWS). The data comprise two full grating scans (using the observing templates SWS01 with scan rate 2 and LWS01 with sampling interval 1) and a set of deeper exposures of selected individual lines (using the SWS02 template). A detailed description of the observing templates can be found in de Graauw et al. (1996) for SWS and Clegg et al. (1996) for LWS. The SWS01 and LWS01 data were reduced from the Auto Analysis Result produced with the ISO pipeline, version 6.0. The reduction was done using ISAP v1.5 and LIA v7.1 and consisted of the removal of cosmic-ray hits and other artifacts and the subsequent averaging and smoothing of the data in each band. The SWS02 data were reduced from Standard Processed Data produced with the ISO pipeline, version 6.0. The SWS Interactive Analysis System was used to remove spikes attributed to ionizedparticle impacts and to align the continua.

Almost all of the emission lines identified in the SWS and LWS spectra have never been observed before and are a valuable extension to the UV and optical spectra of this nebula. This is especially the case since various lines of highly ionized atoms, which are impossible to observe from the ground, have been detected. These lines allow a more reliable determination of the stellar temperature than was hitherto possible. To emphasize this point we show the SWS02 detection of the [Ne vI] $7.65 \mu \mathrm{m}$ line in Figure 3, which is now the highest excitation line ever observed in the spectrum of NGC 6445 .

\subsection{Literature Data}

The optical spectrum was supplemented with data in the range 372.6-396.8 $\mathrm{nm}$ taken from Aller et al. (1973). These data were obtained at three different positions in the nebula

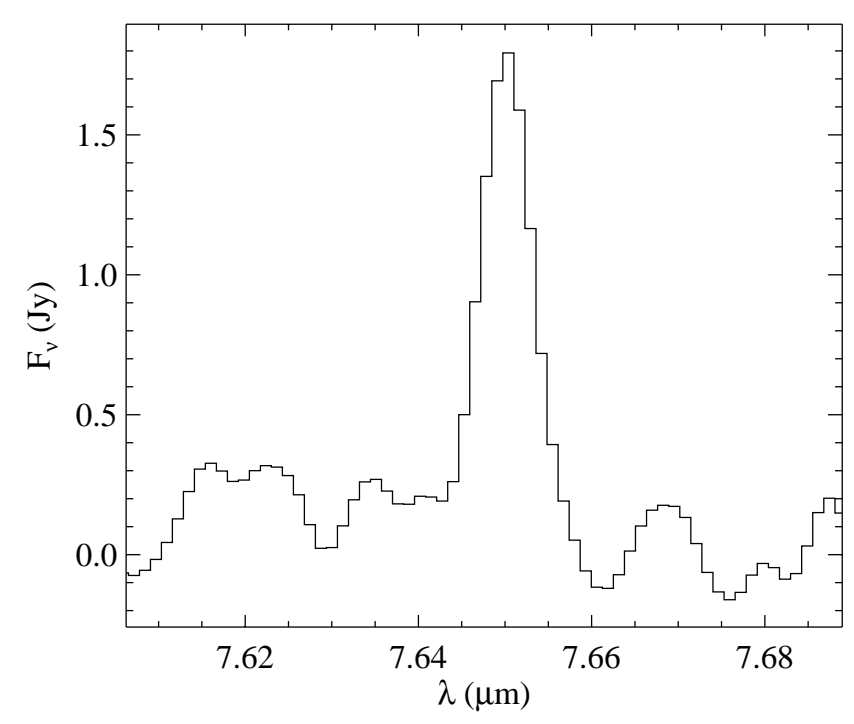

FIG. 3.-Detection of [Ne vI] in NGC 6445 and subsequently averaged, so they are a fair approximation for the integrated flux. The [Ne v] $342.6 \mathrm{~nm}$ flux was taken from Rowlands et al. (1993). These observations used a $48^{\prime \prime}$ aperture, which should be large enough to encompass nearly all of the [Ne v] flux. The adopted fluxes are listed in Table 3.

An $\mathrm{H} \alpha+[\mathrm{N}$ II] image of NGC 6445 was obtained by Schwarz, Corradi, \& Melnick (1992) and is shown in Figure 1. From this image it is clear that the ionized region of NGC 6445 comprises not only the high surface brightness region that is readily observable but also low surface brightness regions that extend much farther. The entire ionized region measures at least $2^{\prime} \times 3^{\prime}$.

The nebula has been observed at radio frequencies on several occasions, both using single-dish telescopes and interferometers. There is a significant difference between the fluxes obtained with the two types of telescopes. This is most likely due to the presence of extended low surface brightness radio emission, which was included in the beam of single dish telescopes but must have been missed in the interferometer observations. The discrepancy between the fluxes cannot be attributed to optical depth effects since modeling of the nebula shows that optical depth effects set in only below $1 \mathrm{GHz}$. Hence it is safe to assume that the radio emission is still optically thin, even at the lowest frequencies observed with the VLA and Parkes. For the total radio flux emitted by the nebula (including low surface brightness regions) we adopt the $14.7 \mathrm{GHz}$ radio flux of 322 mJy obtained with the Parkes $64 \mathrm{~m}$ radio telescope (Milne \& Aller 1982).

The distance of NGC 6445 is not accurately known. Sabbadin (1986a) lists two extinction distances, 2.0 and $2.5 \mathrm{kpc}$. Various other determinations that have been published in the literature are usually lower. We will adopt the statistical distance based on an empirical relation between the surface brightness temperature and the radius of the nebula discussed by Van de Steene \& Zijlstra (1995). In order to obtain a distance with this method, we will need an angular diameter and the radio flux contained within that radius. The Parkes data are not suitable for this purpose, since they contain emission from low surface brightness regions and the angular diameter of that region is not well defined. As an alternative we will use the VLA $1.46 \mathrm{GHz}$ data presented in Phillips \& Mampaso (1988). They obtained a radio flux of $243 \mathrm{mJy}$ and an angular extent of the high surface brightness region of $30^{\prime \prime} .7 \times 40^{\prime \prime} 2$. We will adopt the resulting geometric mean of $35^{\prime \prime} 1$ as the angular diameter. It is consistent to use these data, and the resulting value for the distance is $1.5 \mathrm{kpc}$. This result is not very sensitive to the adopted radio flux density $S_{v}$ since it scales as $S_{v}^{-0.35}$. Using the Parkes flux instead of the VLA flux would yield a result that is only $15 \%$ lower.

Using the data presented above we find that the dynamical age $t_{\mathrm{dyn}}$ of the nebula is $t_{\mathrm{dyn}}=\Theta_{r} D / v_{\mathrm{exp}}=3300 \mathrm{yr}$. Here $\Theta_{r}$ stands for the angular radius of the nebula, $D$ for the distance, and $v_{\text {exp }}$ for the expansion velocity.

\section{APERTURE CORRECTIONS}

Although all of the spectral data we present have been taken with large apertures, none of them encompass all of the nebula. This is especially the case for the $I U E$ and SWS observations, in which significant parts of the high surface brightness regions have been missed. Therefore aperture corrections will be necessary to use these data for modeling 
the nebula. The apertures are overplotted on an image of NGC 6445 in Figure 1.

The IUE-SWP large aperture measures 9."07 × 21".65 (IUE1 and 2). SWS Data in the ranges $2.38-12.0 \mu \mathrm{m}$ were taken with an aperture of $14^{\prime \prime} \times 20^{\prime \prime}$ (SWS-A), 12.0-27.6 $\mu \mathrm{m}$ with an aperture of 14" $\times 27^{\prime \prime}$ (SWS-B), and 29.5-45.2 $\mu \mathrm{m}$ with an aperture of $20^{\prime \prime} \times 33^{\prime \prime}$ (SWS-C). Data in the range 27.6-29.5 $\mu \mathrm{m}$ were taken with a $20^{\prime \prime} \times 27^{\prime \prime}$ aperture. However, since there are no observed lines in this range, this aperture will be ignored in the subsequent discussion. To obtain aperture correction factors for the IUE and SWS data, the total flux was integrated inside each of the aperture boxes and compared to the total flux emitted by the nebula. For this we used the $\mathrm{H} \alpha+[\mathrm{N} \mathrm{II}]$ image of Schwarz et al. (1992). We obtained the following aperture correction factors, which we will adopt in the remainder of the paper: for IUE1, 15.43; for IUE2, 9.98; for SWS-A, 5.27; for SWS-B, 4.65; and for SWS-C, 3.45.

The correction factors for the $I U E$ data are large since this instrument measured only a small region in the center of the nebula, missing most of the highest surface brightness regions. This correction could have been overestimated because of any of the following three effects. First, errors of a few arcseconds in the satellite pointing could cause more flux to enter the aperture. Second, light from the brightest regions just outside the aperture could have been scattered into the line of sight by the intervening ISM. Third, the lines $I U E$ detected could come from highly ionized species that could be more centrally concentrated than the $\mathrm{H} \mathrm{I}+[\mathrm{N}$ II $]$ emission. The IUE fluxes should therefore be considered more uncertain than any of the other data.

The fact that the SWS apertures are centered on a surface brightness peak assures that the first two effects will have less influence on the correction factors for these data. Ionization stratification could, however, still be important. To study this further, we inspected the [O III] image taken by Schwarz et al. (1992). The correction factors for the SWS data derived from this image agree with the ones listed above at the $10 \%$ level. This can be explained by the fact that the positioning of the apertures (which include both ionized and neutral material) favors a small effect on the correction factors. We therefore conclude that the aperture corrections for the SWS data are fairly reliable.

The apertures for the optical and ISO LWS observations are much bigger than the IUE and ISO SWS apertures and cover all of the high surface brightness regions of the nebula. We will not apply any aperture corrections to these data.

\section{INTERSTELLAR REDDENING}

The values for the interstellar extinction toward NGC 6445 quoted in the literature vary considerably and should therefore be considered uncertain. In view of this fact and also because we have spectra covering a wide range of wavelengths, a careful analysis of the data is warranted in order to obtain an accurate extinction curve. Such an analysis is necessarily an iterative process: in order to obtain an extinction curve we need estimates for the electron temperature and density, which in turn can be determined accurately only after an extinction correction has been applied. Luckily the dependence on these quantities is only moderate to weak and simple estimates are sufficient. In the following paragraphs we adopt values for the electron temperature and density that do not agree precisely with each other or with the final values, which will be derived further on in the paper. We are however convinced that the assumed values are sufficiently accurate to ensure that the errors introduced by these assumptions are far less than any other source of error.

We will start the analysis by comparing the radio and $\mathrm{H} \beta$ fluxes. Since we estimate that the optical aperture encompasses roughly $90 \%$ of the total flux emitted by the nebula, it is appropriate to use the Parkes $14.7 \mathrm{GHz}$ flux of $322 \pm 18 \mathrm{mJy}$ (Milne \& Aller 1982) for this comparison. Substituting this value in equation (IV-26) of Pottasch (1984) and adopting $Y=1.23 \pm 0.05$ and an average electron temperature of $T_{e}=13.8 \pm 1.3 \mathrm{kK}$ (obtained from a preliminary analysis of the [O III] lines), we were able to obtain an estimate for the dereddened (intrinsic) $\mathrm{H} \beta$ flux: $\log \left[F(\mathrm{H} \beta) /\left(\mathrm{W} \mathrm{m}^{-2}\right)\right]=-13.06 \pm 0.04$. When we compare this with the observed $\mathrm{H} \beta$ flux of $\log [F(\mathrm{H} \beta) /(\mathrm{W}$ $\left.\left.\mathrm{m}^{-2}\right)\right]=-14.12 \pm 0.04$, we find that $c(\mathrm{H} \beta)=1.06 \pm 0.06$ dex, or alternatively $A(486.1 \mathrm{~nm})=2.64 \mathrm{mag}$.

We will now determine the shape of the extinction curve for our spectra. The relative line strengths in the recombination spectrum of $\mathrm{H} \mathrm{I}$ and $\mathrm{He}$ II depend very little on electron temperature and density and can be determined using case B theory. This allows us to determine the absolute extinction at various other wavelengths using the value for $A(486.1 \mathrm{~nm})$. We adopt the intensity ratios calculated by Storey \& Hummer (1995), assuming an electron density of $10^{3} \mathrm{~cm}^{-3}$ and an electron temperature of $12.5 \mathrm{kK}$ and 15 $\mathrm{kK}$ for the $\mathrm{H} \mathrm{I}-$ and $\mathrm{He}$ II-emitting regions, respectively. These assumptions introduce an error of approximately $1 \%$ or less in the case B line ratios and are therefore well justified. The results are shown in Table 2. The case B ratios are normalized to $\mathrm{H}$ I $486.1 \mathrm{~nm}$ and $\mathrm{He}$ II $468.6 \mathrm{~nm}$ for the hydrogen and helium spectra, respectively. In order to obtain a value for the extinction at $468.6 \mathrm{~nm}$, a fit was made to the observed extinctions for the hydrogen lines only, using a standard ISM extinction law (Cardelli, Clayton, \& Mathis 1989) and adopting a ratio of the total to specific extinction $R_{V} \equiv A(V) / E(B-V)=3.3$. This allowed us to obtain an absolute flux of the $\mathrm{He}$ II $468.6 \mathrm{~nm}$ line and to include the other He II lines in the analysis. The results are shown in Table 2 and Figure 4.

To obtain an extinction curve, we made a least-squares fit of the law given by Cardelli et al. (1989) to these data, using the ratio of total to selective extinction, $R_{V}$, and the selective

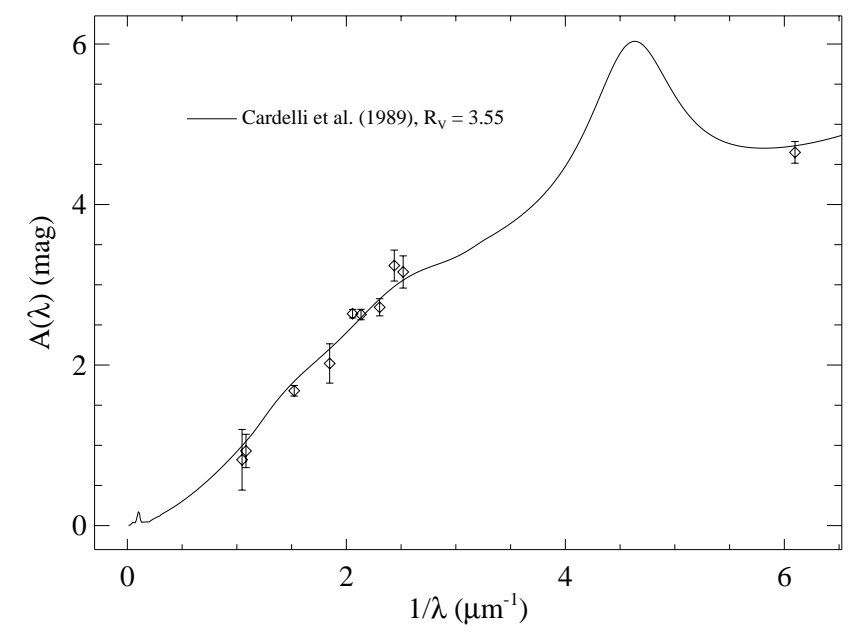

FIG. 4.-Extinction law for NGC 6445 
TABLE 2

EMPIRICALly Determined EXTINCTION VALUES

\begin{tabular}{|c|c|c|c|c|c|c|}
\hline Spectrum & $\begin{array}{c}\lambda \\
(\mathrm{nm})\end{array}$ & $\begin{array}{l}\text { Observed Flux } \\
\left(10^{-14} \mathrm{~W} \mathrm{~m}^{-2}\right)\end{array}$ & $\begin{array}{l}\text { Case B } \\
\text { Ratio }\end{array}$ & $\begin{array}{l}\text { Predicted Flux } \\
\left(10^{-14} \mathrm{~W} \mathrm{~m}^{-2}\right)\end{array}$ & $\begin{array}{c}1 / \lambda \\
\left(\mu \mathrm{m}^{-1}\right)\end{array}$ & $\begin{array}{c}A(\lambda) \\
\text { (mag) }\end{array}$ \\
\hline H I $\ldots \ldots$. & 954.6 & 0.146 & 0.0357 & 0.311 & 1.048 & 0.82 \\
\hline H I $\ldots . . .$. & 922.9 & 0.0922 & 0.0249 & 0.216 & 1.084 & 0.93 \\
\hline H I ....... & 656.3 & 5.19 & 2.8158 & 24.5 & 1.524 & 1.68 \\
\hline $\mathrm{He}$ II ....... & 541.2 & 0.0508 & 0.0787 & 0.327 & 1.848 & 2.02 \\
\hline H I ...... & 486.1 & 0.762 & 1.0000 & 8.70 & 2.057 & 2.64 \\
\hline He il ...... & 468.6 & 0.368 & 1.0000 & 4.16 & 2.134 & 2.63 \\
\hline H I $\ldots . . .$. & 434.0 & 0.334 & 0.4713 & 4.10 & 2.304 & 2.72 \\
\hline H I $\ldots . . .$. & 410.2 & 0.115 & 0.2612 & 2.27 & 2.438 & 3.24 \\
\hline H I $\ldots . . .$. & 397.0 & 0.0762 & 0.1605 & 1.40 & 2.519 & 3.16 \\
\hline He II...... & 164.0 & 0.399 & 6.9882 & 29.0 & 6.098 & 4.65 \\
\hline
\end{tabular}

extinction, $E(B-V)$, as free parameters. The result was $R_{V}=3.55$ and $E(B-V)=0.61$, which is equivalent to $c(\mathrm{H} \beta)=0.99$ dex. We have composed an extinction law using the results of Cardelli et al. (1989) with $R_{V}=3.55$ for wavelengths between 0.1 and $3.33 \mu \mathrm{m}$, Rieke \& Lebofsky (1985) for those between 3.33 and $13 \mu \mathrm{m}$, and Mathis (1990) for wavelengths longward of $13 \mu \mathrm{m}$. The extinction law that is obtained this way is plotted in Figure 4 and was used to deredden all observed fluxes.

Altering the value of $R_{V}$ has a strong effect on the UV part of the extinction curve but leaves the optical and infrared parts virtually unchanged. Hence the fact that we use an $R_{V}$ that deviates from the canonical value $R_{V}=3.1$ is important for the dereddening of the UV line fluxes and ensures that the case B line ratios for He II are obeyed. On the other hand, if we had used an extinction law with $R_{V}=$ 3.1 , this would have made a difference of only $10 \%$ or less for the optical and infrared line intensities. In other words, in cases where only optical and/or infrared data are available, the value of $R_{V}$ cannot be determined from these data and the assumption $R_{V}=3.1$ is appropriate. However, in cases where UV data is included, $R_{V}$ should be treated as a free parameter to ensure a proper dereddening.

The results of the dereddening can be found in Table 3, where we list the fluxes and the dereddened intensities relative to $\mathrm{H} \beta$ for all the lines used in this paper. Aperture correction factors have been applied to the dereddened intensities, but not to the observed fluxes (except for the combined IUE spectra). The error bars quoted in Table 3 reflect purely the uncertainty in the measured flux and are therefore a measure for the quality of the spectrum. They do not contain contributions for the uncertainties in the absolute flux calibration, aperture correction, or reddening correction. However, estimates for these uncertainties where quadratically added to make the photoionization model discussed in $\S 8$.

\section{REMARKS ON THE OBSERVED SPECTRUM}

The observed flux for the [O III] $88.4 \mu \mathrm{m}$ line is more than a factor of 5 higher than the $3 \sigma$ upper limit of $2 \times 10^{-14} \mathrm{~W}$ $\mathrm{m}^{-2}$, which was previously determined by Dinerstein, Lester, \& Werner (1985). This large discrepancy cannot be explained by aperture effects since both observations encompass essentially all of the nebula. It also seems very unlikely that it is caused by variability in the spectrum since there is no evidence that would support this assumption. Comparison of our [O III] $500.7 \mathrm{~nm}$ flux with the ones published in O'Dell (1963) and Aller et al. (1973) shows that they all are in good agreement. Our flux is only $0.06 \mathrm{dex}$ higher than the older measurements, making it very unlikely that the infrared [O III] spectrum has undergone large changes over the past $35 \mathrm{yr}$. An explanation for this conundrum is therefore not apparent to us.

The intensity $I(\mathrm{H} \alpha)=363$ is surprisingly large and is not expected according to case B theory. A possible explanation could be provided by the fact that the overall shape of the extinction curve can differ from the mean law for different lines of sight (Cardelli et al. 1989). Hubble Space Telescope observations could be used to investigate the extinction law further.

Another explanation could be provided by the fact that the outer halo of NGC 6445 is currently recombining (Tylenda 1986), which would cause the plasma to have an electron temperature well below what is expected for an ionized region and would alter the case B line ratios. From our discussion in $\S 8$ it will become clear that the central star is currently evolving on the cooling track in the Hertzsprung-Russell diagram, which implies that it has undergone a considerable drop in luminosity in recent history. This evolution could have caused the ionized region to shrink. Therefore it is possible that the faint surface brightness regions are actually the afterglow of regions that did not have sufficient time to recombine. The recombination timescale for hydrogen is approximately $1230 T_{4}^{0.83} n_{2}^{-1} \mathrm{yr}$, where $T_{4}$ is the electron temperature in units of $10^{4} \mathrm{~K}$ and $n_{2}$ is the electron density in units of $10^{2}$ $\mathrm{cm}^{-3}$ (using Storey \& Hummer 1995). This timescale could very well be longer than the evolutionary timescale of the central star, depending on the density in the outer halo. However, the timescale for recombination from $\mathrm{O}^{2+}$ to $\mathrm{O}^{+}$ is only $86 T_{4}^{0.52} n_{2}^{-1} \mathrm{yr}$ (using the atomic data referenced in CLOUDY 90.05). This would imply that in the outermost regions of the halo, where the gas supposedly had the longest time to recombine, one would not expect [O III] $500.7 \mathrm{~nm}$ emission to be visible. Inspection of the [O III] image taken by Schwarz et al. (1992), however, reveals that such emissions are present. The outermost regions in the northeast and southwest directions are clearly visible in both the $\mathrm{H} \alpha+[\mathrm{N}$ II $]$ and the $[\mathrm{O}$ III $]$ image, indicating that they are still being photoionized. Some regions in the southeast and northwest directions, however, do appear much fainter in [O III], and it is plausible that recombination is going on there. To illustrate this point, we show in Figure 5 the ratio of the [O III] image to the $\mathrm{H} \alpha+[\mathrm{N} \mathrm{II}]$ image. It can be interpreted as a measure for the level of excitation, where white indicates high excitation. The scale 
TABLE 3

ObSERVEd LINe FluXES AND DEREDDENED INTENSITIES FOR NGC 6445

\begin{tabular}{|c|c|c|c|c|c|}
\hline Spectrum & $\begin{array}{c}\lambda \\
(\mathrm{nm})\end{array}$ & $\begin{array}{c}\text { Flux } \\
\left(10^{-14} \mathrm{~W} \mathrm{~m}^{-2}\right)\end{array}$ & $\begin{array}{c}\sigma \\
(\%)\end{array}$ & $\begin{array}{c}\text { Intensity } \\
I(\mathrm{H} \beta)=100\end{array}$ & Comment \\
\hline \multirow[t]{3}{*}{$\mathrm{C}$ iv $\ldots \ldots \ldots$} & 154.9 & 0.015 & 17.1 & $\ldots$ & IUE1 \\
\hline & & 0.026 & 32.1 & $\ldots$ & IUE2 \\
\hline & & $0.247:^{\mathrm{a}}$ & 16.2 & $283 .:^{\mathrm{a}}$ & Combined \\
\hline \multirow[t]{3}{*}{ He II ......... } & 164.0 & 0.024 & 13.7 & $\ldots$ & IUE1 \\
\hline & & 0.040 & 17.5 & $\ldots$ & IUE2 \\
\hline & & $0.399:^{a}$ & 13.2 & $416 .:^{a}$ & Combined \\
\hline \multirow[t]{3}{*}{$\mathrm{C}$ III] $\ldots \ldots \ldots$} & 190.9 & 0.037 & 5.6 & $\ldots$ & IUE1 \\
\hline & & 0.053 & 8.7 & $\ldots$ & IUE2 \\
\hline & & $0.556:^{\mathrm{a}}$ & 5.7 & $712 .:^{a}$ & Combined \\
\hline$[\mathrm{Ne} v] \ldots \ldots$ & 342.6 & 0.050 & 20.0 & 13.9 & \\
\hline$[\mathrm{O} \mathrm{II}] \ldots \ldots \ldots$ & 372.6 & 0.838 & $\ldots$ & 209. & \\
\hline$[\mathrm{O}$ II $] \ldots \ldots \ldots$ & 372.9 & 0.769 & $\ldots$ & 192. & \\
\hline$[\mathrm{Ne}$ III $] \ldots . .$. & 386.9 & 0.983 & $\ldots$ & 231. & \\
\hline$[\mathrm{Ne}$ III $] \ldots . .$. & 396.8 & 0.312 & $\ldots$ & 69.8 & \\
\hline $\mathrm{H}_{\text {I }} \ldots . . . . . .$. & 397.0 & 0.076 & 20.3 & 17.0 & \\
\hline$[\mathrm{S}$ II $] \ldots \ldots \ldots$ & 407.2 & 0.055 & 51.2 & 11.6 & \\
\hline $\mathrm{H}_{\mathrm{I}} \ldots . . . \ldots .$. & 410.2 & 0.115 & 19.4 & 23.9 & \\
\hline H I .......... & 434.0 & 0.334 & 10.4 & 60.0 & \\
\hline$[\mathrm{O}$ III $] \ldots \ldots$ & 436.3 & 0.123 & 24.6 & 21.8 & \\
\hline He I ........... & 447.1 & 0.058 & 38.7 & 9.6 & \\
\hline He II ........ & 468.6 & 0.368 & 6.2 & 53.3 & \\
\hline$[\mathrm{Ar} \mathrm{IV}] \ldots \ldots$. & 471.1 & 0.044 & 36.2 & 6.2 & \\
\hline$[\mathrm{Ar} \mathrm{IV}] \ldots \ldots .$. & 474.0 & 0.030 & 59.7 & 4.2 & \\
\hline $\mathrm{H}_{\text {I }} \ldots \ldots \ldots \ldots$ & 486.1 & 0.762 & 5.0 & 100. & \\
\hline$[\mathrm{O}$ III $] \ldots \ldots$ & 495.9 & 3.84 & 4.6 & 479. & \\
\hline$[\mathrm{O} \mathrm{III}] \ldots \ldots .$. & 500.7 & 12.2 & 6.5 & 1490. & \\
\hline$\left[\mathrm{N}_{\mathrm{I}}\right] \ldots \ldots \ldots$ & 520.0 & 0.145 & 18.0 & 16.0 & \\
\hline He II ......... & 541.2 & 0.051 & 25.3 & 5.1 & \\
\hline$[\mathrm{N}$ II $] \ldots \ldots .$. & 575.5 & 0.112 & 12.8 & 10.0 & \\
\hline He I ........... & 587.6 & 0.178 & 8.2 & 15.3 & \\
\hline$\left[\mathrm{O}_{\mathrm{I}}\right] \ldots \ldots \ldots$ & 630.0 & 0.503 & 3.6 & 37.9 & \\
\hline$[\mathrm{S}$ III $] \ldots \ldots \ldots$ & 631.2 & 0.037 & 21.2 & 2.8 & \\
\hline$\left[\mathrm{O}_{\mathrm{I}}\right] \ldots \ldots \ldots$ & 636.4 & 0.153 & 4.6 & 11.3 & \\
\hline$[\mathrm{N}$ II $] \ldots \ldots .$. & 654.8 & 3.19 & 8.3 & 224. & \\
\hline H I ............ & 656.3 & 5.19 & 6.3 & 363. & \\
\hline$[\mathrm{N}$ II $] \ldots \ldots .$. & 658.4 & 9.51 & 4.6 & 661. & \\
\hline He I ........... & 667.8 & 0.058 & 14.1 & 3.9 & \\
\hline$[\mathrm{S}$ II $] \ldots \ldots \ldots$ & 671.6 & 0.803 & 5.0 & 53.6 & \\
\hline$[\mathrm{S}$ II $] \ldots \ldots \ldots$ & 673.1 & 0.755 & 5.2 & 50.3 & \\
\hline$[\mathrm{Ar} \mathrm{v}] \ldots \ldots .$. & 700.6 & $<0.052$ & $\ldots$ & $<3.2$ & \\
\hline He I .......... & 706.5 & 0.056 & 7.7 & 3.4 & \\
\hline$[\mathrm{Ar} \mathrm{III}] \ldots \ldots$ & 713.6 & 0.749 & 3.1 & 44.2 & \\
\hline$[\mathrm{Ca}$ II $] \ldots \ldots .$. & 729.1 & $<0.017$ & $\ldots$ & $<1.0$ & \\
\hline$[\mathrm{O}$ II $] \ldots \ldots \ldots$ & 731.9 & 0.221 & 9.2 & 12.4 & \\
\hline$[\mathrm{O} \quad \mathrm{II}] \ldots \ldots .$. & 733.0 & 0.185 & 10.2 & 10.3 & \\
\hline$[\mathrm{Ar} \mathrm{IV}] \ldots \ldots .$. & 733.2 & $<0.019$ & $\ldots$ & $<1.0$ & \\
\hline$[\mathrm{Ar} \mathrm{III}] \ldots \ldots$. & 775.1 & 0.171 & 12.8 & 8.5 & \\
\hline$[\mathrm{Ca}$ III $] \ldots . . .$. & 849.8 & $<0.033$ & $\ldots$ & $<1.3$ & \\
\hline$[\mathrm{Ca} \quad \mathrm{II}] \ldots . .$. & 854.2 & $<0.035$ & $\ldots$ & $<1.4$ & \\
\hline$[\mathrm{S}$ III $] \ldots \ldots . .$. & 906.9 & 1.11 & 3.7 & 39.9 & \\
\hline H I ............ & 922.9 & 0.092 & 21.1 & 3.2 & \\
\hline$[\mathrm{S}$ III $] \ldots \ldots .$. & 953.2 & 2.73 & 7.7 & 91.1 & \\
\hline H I ........... & 954.6 & 0.146 & 41.6 & 4.9 & \\
\hline $\mathrm{H}_{\text {I }} \ldots \ldots \ldots . .$. & 4052 & 0.094 & 3.5 & $7.2^{\mathrm{a}}$ & SWS02 \\
\hline$[\mathrm{Mg}$ IV $] \ldots \ldots$ & 4487 & 0.074 & 8.6 & $5.5^{\mathrm{a}}$ & SWS02 \\
\hline$[\mathrm{Ar} \mathrm{VI}] \ldots \ldots \ldots$ & 4530 & $<0.051$ & $\ldots$ & $<3.8^{\mathrm{a}}$ & SWS02 \\
\hline$[\mathrm{Mg} \mathrm{v}] \ldots \ldots$ & 5610 & $<0.142$ & $\ldots$ & $<10.4^{\mathrm{a}}$ & SWS02 \\
\hline$[\mathrm{Ne} \mathrm{vI}] \ldots \ldots$ & 7652 & 0.052 & 10.3 & $3.8^{\mathrm{a}}$ & SWS02 \\
\hline$[\mathrm{Ar}$ III $] \ldots \ldots$. & 8991 & 0.339 & 14.9 & $27.4^{\mathrm{a}}$ & SWS01 \\
\hline$[\mathrm{S}$ IV $] \ldots \ldots \ldots$ & 10510 & 0.954 & 9.7 & $77.0^{\mathrm{a}}$ & SWS01 \\
\hline$[\mathrm{Ne}$ II $] \ldots \ldots .$. & 12814 & 0.377 & 1.6 & $24.7^{\mathrm{a}}$ & SWS02 \\
\hline$[\mathrm{Mg} \mathrm{v}] \ldots \ldots$ & 13521 & $<0.032$ & $\ldots$ & $<2.1^{\mathrm{a}}$ & SWS02 \\
\hline$[\mathrm{Ne} v] \ldots \ldots$. & 14322 & 1.64 & 1.8 & $105^{\mathrm{a}}$ & SWS02 \\
\hline$[\mathrm{Ne}$ III $] \ldots .$. & 15555 & 4.05 & 1.0 & $259 .^{\mathrm{a}}$ & SWS02 \\
\hline
\end{tabular}


TABLE 3-Continued

\begin{tabular}{|c|c|c|c|c|c|}
\hline Spectrum & $\begin{array}{c}\lambda \\
(\mathrm{nm})\end{array}$ & $\begin{array}{c}\text { Flux } \\
\left(10^{-14} \mathrm{~W} \mathrm{~m}^{-2}\right)\end{array}$ & $\begin{array}{c}\sigma \\
(\%)\end{array}$ & $\begin{array}{c}\text { Intensity } \\
I(\mathrm{H} \beta)=100\end{array}$ & Comment \\
\hline$[\mathrm{S}$ III $] \ldots \ldots$. & 18713 & 0.796 & 11.9 & $51.5^{\mathrm{a}}$ & SWS01 \\
\hline$[$ Ar III $] \ldots . .$. & 21829 & $<0.276$ & $\ldots$ & $<17.7^{\mathrm{a}}$ & SWS01 \\
\hline$[\mathrm{Ne} v\rceil \ldots \ldots$ & 24318 & 1.98 & 1.0 & $126^{\mathrm{a}}$ & SWS02 \\
\hline$[\mathrm{O}$ IV $] \ldots \ldots$. & 25890 & 10.8 & 1.2 & $686^{\mathrm{a}}$ & SWS02 \\
\hline$[\mathrm{S}$ III $] \ldots \ldots .$. & 33481 & 1.44 & 14.3 & $67.0^{\mathrm{a}}$ & SWS01 \\
\hline$[\mathrm{Si}$ II $] \ldots \ldots .$. & 34815 & 0.488 & 26.4 & $22.6^{\mathrm{a}}$ & SWS01 \\
\hline$[\mathrm{Ne}$ III $] \ldots . .$. & 36014 & 0.360 & 5.4 & $16.7^{\mathrm{a}}$ & SWS02 \\
\hline$[\mathrm{O}$ III $] \ldots \ldots$ & 51815 & 19.0 & 1.8 & 255. & \\
\hline$[\mathrm{N}$ III $] \ldots \ldots .$. & 57340 & 6.30 & 1.6 & 84.4 & \\
\hline \multirow[t]{2}{*}[\mathrm{O}_{\mathrm{I}}]{$\ldots \ldots \ldots$} & $63185^{b}$ & 4.71 & 1.4 & 63.0 & \\
\hline & & 0.124 & 23.4 & 1.7 & Off source \\
\hline$[\mathrm{O} \mathrm{III}] \ldots \ldots$. & 88356 & 10.9 & 1.4 & 146. & \\
\hline$[\mathrm{N}$ II $] \ldots \ldots .$. & 121760 & 0.402 & 2.9 & 5.4 & \\
\hline$\left[\begin{array}{ll}\mathrm{I}\end{array}\right] \ldots \ldots \ldots$ & $145535^{b}$ & 0.235 & 2.3 & 3.1 & \\
\hline \multirow[t]{2}{*}[\mathrm{C}\text{II}]{$\ldots \ldots . . . .}$. & 157680 & 0.894 & 1.7 & $12.0^{\mathrm{c}}$ & \\
\hline & & 0.241 & 14.0 & $3.2^{\mathrm{c}}$ & Off source \\
\hline
\end{tabular}

NoTE.-Where appropriate $3 \sigma$ upper limits are given.

${ }^{a}$ An aperture correction factor has been applied.

b This line was not used to constrain the photoionization model.

c The off-source flux was subtracted from the on-source flux to constrain the modeling.

and positioning of the image is identical to Figure 1. In order to increase the signal-to-noise ratio in the low surface brightness regions, the original images were smoothed with a Gaussian with a FWHM of 2 pixels before they were divided. The resulting image clearly shows the complex structure of the nebula. The darkest regions in the image are either low-excitation regions that are close to ionization fronts or unilluminated regions where recombination is taking place. The image clearly shows that especially the northeast part of the halo is still highly excited. Hence the simple view in which the entire halo is recombining cannot be correct. Because of the evolutionary status of the central

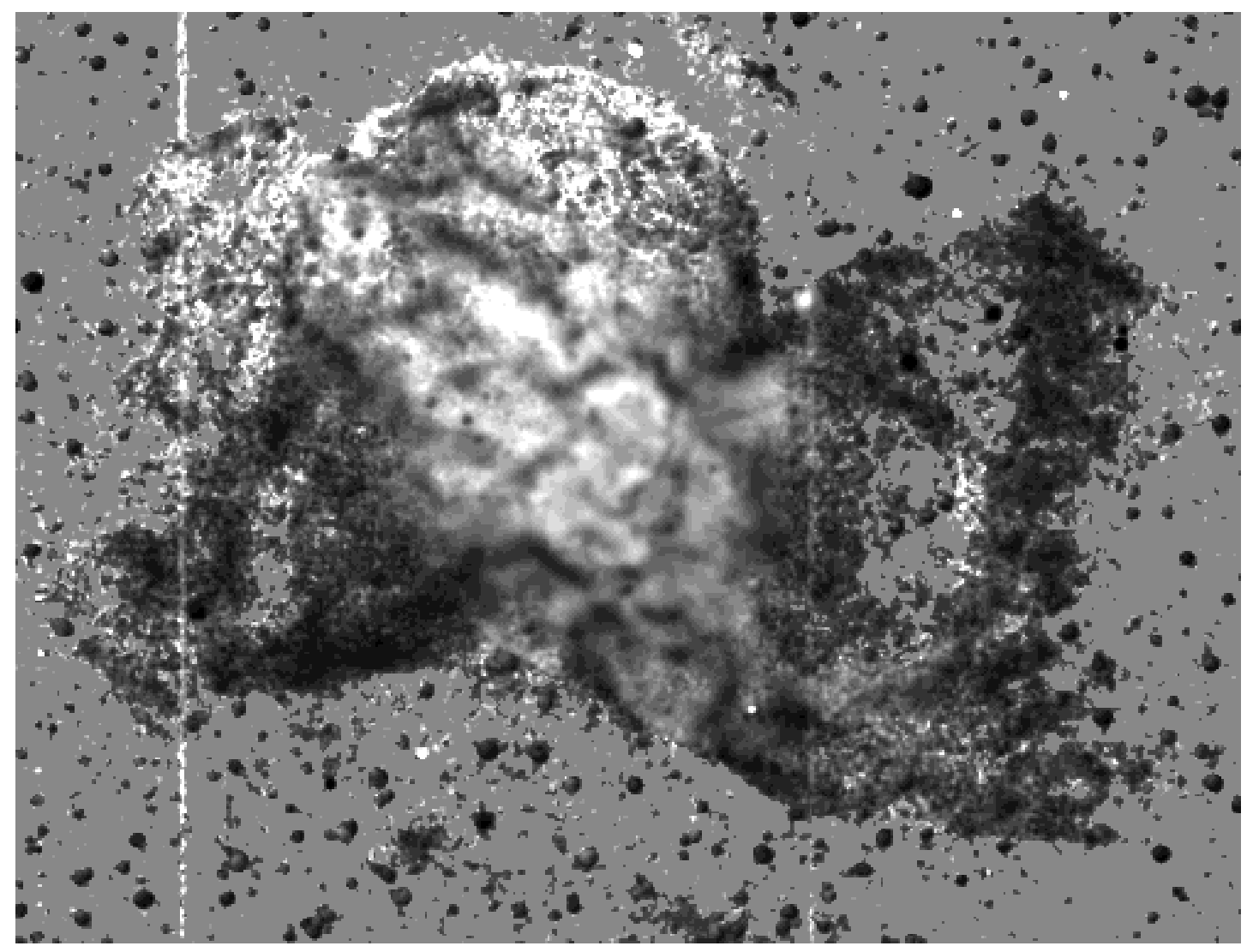

FIG. 5.-Ratio of the [O III] to $\mathrm{H} \alpha+[\mathrm{N} \mathrm{II}]$ image of NGC 6445 (Schwarz et al. 1992). Dark regions indicate low excitation and are interpreted as regions near an ionization front or unilluminated regions that are currently recombining. 
star, it is likely, however, that recombination is taking place to some degree. This is the case for the southeast and northwest regions, but probably near every ionization front as well. We cannot obtain an accurate estimate for the emission measure of these regions, but it seems likely it is insufficient to have a large effect on the integrated hydrogen spectrum, at least within the optical aperture. On the other hand, recombination is expected to have a profound effect on the conditions in the photodissociation region (PDR). This is because the region that is now the PDR was in recent history part of the ionized region and therefore still has a "memory" of those conditions. In particular, its energy content will be higher than expected from equilibrium calculations, and therefore the electron temperature will also be higher. This implies that CLOUDY may not be able to correctly predict the PDR emissions for this nebula.

The fact that ionization fronts can be seen in virtually every direction in Figure 5 is a strong support for the assumption that the nebula is ionization bounded and that virtually no Lyman continuum photons can escape it. That the nebula is ionization bounded is possible because the central star is on the cooling track and emits far fewer ionizing photons than when it was still on the horizontal track. The fact that ionization fronts can clearly be seen in the outer halo proves that the halo cannot be a reflection nebula.

\section{PLASMA DIAGNOSTICS AND THE $t^{2}$ EFFECT}

Accurate abundance determinations are hampered by the so-called $t^{2}$ problem (Peimbert 1967). This problem is probably one of the most important in present day PN research and is subject to much debate. The $t^{2}$ parameter is a measure of the electron temperature fluctuations in the ionized region of the nebula. Models do not predict such fluctuations, but the limited amount of observational material gathered so far seems to contradict this. The $t^{2}$ problem can be investigated by comparing the strength of infrared lines with optical lines. The emissivities of the farinfrared fine-structure lines have a very different dependence on electron temperature and densitythan the optical lines (Simpson 1975; Watson \& Storey 1980). The infrared line strengths are virtually insensitive to $T_{e}$ because of the very small energy of their upper levels, whereas the optical lines originate from higher energy levels and have strong dependence on $T_{e}$. Fluctuations in $T_{e}$ increase the strength of emission lines, and this increase will be larger for lines originating from higher energy levels (e.g., for optical lines). Hence the $t^{2}$ effect can be measured by comparing electron temperatures determined from optical and infrared diagnostic lines.

Using the dereddened line intensities, we are able to derive values for the electron temperature and density using various diagnostic ratios. The results are listed in Table 4. The results for the optical density diagnostics ([S II] and $\left[\begin{array}{ll}\mathrm{O} & \mathrm{II}\end{array}\right]$ ) were obtained with the package NEBULAR in IRAF (Shaw \& Dufour 1995). All other diagnostics were obtained using our own five-level model atom, with collision strengths taken from Lennon \& Burke (1994) for [N II], [O III], and [Ne v]; Butler \& Zeippen (1994) for [Ne III]; and Galavis, Mendoza, \& Zeippen (1995) for [S III]. The transition probabilities were taken from Galavis, Mendoza, \& Zeippen (1997) for [N II], [O III], [Ne III], and [Ne v]; and Mendoza \& Zeippen (1982) for [S III].

The results for the various temperature diagnostics show quite a large spread, which can in part be explained by temperature stratification in the nebula. However, one also notices that the infrared temperature diagnostics seem to give systematically lower results than the optical diagnostics. This could point to a $t^{2}$ effect, although the evidence is not convincing. The temperatures we derived indicate the following values: $t^{2}[\mathrm{O} \mathrm{III}]=0.065 \pm 0.053$ and $t^{2}[\mathrm{~S} \mathrm{III}]=0.168 \pm 0.148$. Hence the error margins are too large to validate the presence of a $t^{2}$ effect.

Other explanations for the difference between the optical and infrared diagnostics are also plausible. First of all, in order to calculate the temperature from the infrared diagnostics, the optical and ISO spectra need to be compared. Hence systematic effects may be introduced by errors in the absolute calibration of either of these spectra and/or by errors in the aperture corrections for the SWS data. The uncertainty introduced by the absolute calibrations has been estimated at $20 \%$, and the uncertainty introduced by the aperture corrections at $15 \%$. These numbers were used to calculate the error margins for the electron temperatures given above, but they may have been underestimated.

Another possible explanation is inaccuracies in the atomic data, in particular the collisional cross sections. Questions concerning the $[\mathrm{Ne} \mathrm{v}]$ data calculated by Lennon \& Burke (1994) have been raised by Oliva, Pasquali, \& Reconditi (1996). However, their argument can be refuted as it is based on an inaccurate flux for the [Ne v] $14.3 \mu \mathrm{m}$ line, as is shown by van Hoof et al. (1997b). Sub-

TABLE 4

Plasma Diagnostics for NGC 6445, Using Various Line Ratios

\begin{tabular}{|c|c|c|c|}
\hline Diagnostic & Ratio & $\begin{array}{c}T_{e} \\
(\mathrm{kK})\end{array}$ & $\begin{array}{c}n_{e} \\
\left(\mathrm{~cm}^{-3}\right)\end{array}$ \\
\hline$[\mathrm{N} \mathrm{II}](654.8+658.4) / 575.5 \ldots \ldots$ & $88 \pm 12$ & $10.4 \pm 0.6$ & $\ldots$ \\
\hline$[\mathrm{S}$ III] $(906.9+953.2) / 631.2 \ldots \ldots$ & $48 \pm 10$ & $11.5 \pm 1.1$ & $\ldots$ \\
\hline$[\mathrm{O} \mathrm{III}](495.9+500.7) / 436.3 \ldots \ldots$ & $90 \pm 23$ & $13.5 \pm 1.2$ & $\ldots$ \\
\hline$[\mathrm{S}$ III $] 953.2 / 18713 \ldots \ldots \ldots \ldots \ldots$ & $1.8 \pm 0.5$ & $8.9 \pm 1.6$ & $\ldots$ \\
\hline [O III] $500.7 / 51815 \ldots \ldots \ldots \ldots \ldots$ & $5.8 \pm 1.2$ & $11.5 \pm 0.9$ & \\
\hline$[\mathrm{Ne}$ III $] 386.9 / 15555 \ldots \ldots \ldots \ldots \ldots$ & $0.89 \pm 0.26$ & $11.0 \pm 0.9$ & \\
\hline$[\mathrm{Ne} \mathrm{v}] 342.6 / 14322 \ldots \ldots \ldots \ldots \ldots$ & $0.13 \pm 0.04$ & $12.0 \pm 0.8$ & $\ldots$ \\
\hline [S II] $671.6 / 673.1 \ldots \ldots \ldots \ldots \ldots . .$. & $1.07 \pm 0.08$ & $\ldots$ & $470 \pm 170$ \\
\hline [O II] $372.6 / 372.9 \ldots \ldots \ldots \ldots \ldots$ & $1.09 \pm 0.11$ & $\ldots$ & $500 \pm 150$ \\
\hline [S III] 18713/33481 ............ & $0.77 \pm 0.14$ & $\ldots$ & $310 \pm 200$ \\
\hline [O III] $51815 / 88356 \ldots \ldots \ldots \ldots .$. & $1.75 \pm 0.18$ & $\ldots$ & $550 \pm 90$ \\
\hline
\end{tabular}


sequent work (van Hoof et al. 2000) has shown that, at least for [Ne v], deviations up to a maximum of $30 \%$ in the data for the transitions in the ground term could be indicated by observations of PNe. For the data in this paper, the difference between the optical and infrared diagnostics can be eliminated by adjusting the collision strengths of the transitions in the ground term at the $30 \%$ level or less. This argument indicates that it is at least conceivable that the differences between the optical and infrared diagnostics are caused by inaccuracies in the collisional cross sections.

The various density diagnostics are in excellent agreement, which suggests that the assumption of constant density is a good approximation for modeling the ionized region of this nebula. The density we find is fairly low for planetary nebulae, which indicates that NGC 6445 is well evolved.

\section{THERMAL GRAIN EMISSION}

The fact that hardly any grain emission is detected in the SWS01 spectrum raises the question whether the inner regions of NGC 6445 could be free of grains, because of either grain destruction or dust-gas separation. The LWS spectrum covers a larger part of the nebula, and grain emission is observed in this spectrum, which indicates that at least part of the grains survived. LWS will have detected the emission from the grains closest to the central star but still may have missed the emission from the cooler grains farther out in the nebula. Before we can study this spectrum, it needs to be corrected for the contribution from the ISM background.

\subsection{Correcting the On-Source Spectrum}

We took an off-source LWS spectrum $3^{\prime}$ north of the nebula at a position angle of roughly $-50^{\circ}$ with respect to the polar axis of the nebula (using the standard convention, in which positive angles indicate a counterclockwise direction). The approximate position of the polar axis is indicated in Figure 1. The off-source spectrum was intended to measure the background continuum and line emission from the ISM. The off-source spectrum is shown in Figure 6. It shows two clearly distinct emission components: one very cool component that becomes detectable only at wavelengths longward of $140 \mu \mathrm{m}$ and a much warmer com-

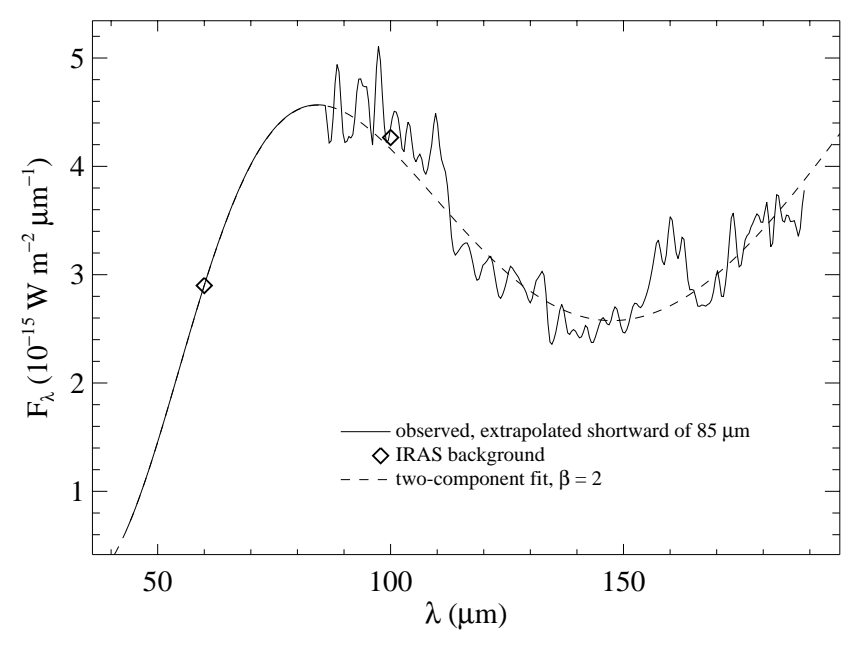

FIG. 6.-Off-source spectrum near NGC 6445 ponent that is visible at shorter wavelengths. The latter is observable as a smooth background in HIRES images made from IRAS 60 and $100 \mu \mathrm{m}$ data. Measurements of this background are shown with a diamond in Figure 6. The flux densities were calculated from the measured surface brightness assuming a circular aperture with a diameter of $84^{\prime \prime}$ for LWS and applying the appropriate AC-DC and color corrections. The excellent agreement of the $100 \mu \mathrm{m}$ point clearly indicates that the warm component of the background emission is associated with cirrus. No significant gradient of the cirrus emission is seen in the vicinity of NGC 6445. The very cold component is not detected in the $100 \mu \mathrm{m}$ HIRES image since its flux is too low compared to the cirrus emission. The cold component is clearly extended (it is seen in both the on-source and off-source spectrum). Estimates for the dust temperature of both components are derived below. The quality of the off-source spectrum was poor at wavelengths shortward of $85 \mu \mathrm{m}$, and therefore an extrapolation was made using the fit discussed below. Hence the extrapolation is primarily based on the background flux measured in the $60 \mu \mathrm{m}$ HIRES image. The ISM contribution is very small compared to the on-source flux at $45 \mu \mathrm{m}$ (less than 2\%). For longer wavelengths the ISM contribution becomes increasingly important: at $85 \mu \mathrm{m}$ the ISM contributes $25 \%$ to the total on-source flux, at $145 \mu \mathrm{m}$ $50 \%$, and at $190 \mu \mathrm{m} 90 \%$.

We need to correct the on-source spectrum for the contribution by the ISM background since this emission would lead to a serious underestimation of the nebular grain temperature. This correction is easily done assuming that the contribution by the ISM is the same in the on-source and off-source spectrum. This assumption is justified, both for the warm and cold components of the ISM emission. From the HIRES images we know that the cirrus emission shows no gradient in the vicinity of the source. The very cold ISM component is the dominant feature at long wavelengths in the on-source spectrum, and this feature is very nicely removed after subtraction. The remaining question is whether grain emission from the outermost regions of the nebula itself could have contributed to the off-source spectrum. This argument leads to estimates of hundreds or even thousands of solar masses for the total nebular mass. This is clearly impossible unless we have seriously overestimated the distance to the nebula. This argument seems very contrived, and we therefore dismiss it. The on-source spectrum was corrected for the contribution of the ISM continuum using the entire spectrum shown in Figure 6. The corrected spectrum is shown in Figure 7. In the off-source spectrum, two emission lines were detected, [O I] $63 \mu \mathrm{m}$ and [C II] 158 $\mu \mathrm{m}$. The fluxes of these lines were subtracted from the onsource flux to constrain our photoionization model of the nebula.

\subsection{Temperature Determination}

To determine the average grain temperature of the ISM background emission and the nebular grain emission, we will use an emission law of the form $F_{v}=c v^{\beta} B_{v}\left(T_{\text {dust }}\right)$, where $c, \beta$, and $T_{\text {dust }}$ are free parameters. Such a simple power-law dependence of the absorption cross section is assumed for astronomical (i.e., amorphous) silicates beyond the $18 \mu \mathrm{m}$ feature and for graphite beyond $35 \mu \mathrm{m}$ (e.g., Draine \& Lee 1984). However, recent $I S O$ observations have shown that crystalline silicates show a rich spectrum of emission features, both in the SWS and LWS wavelength range (e.g., 


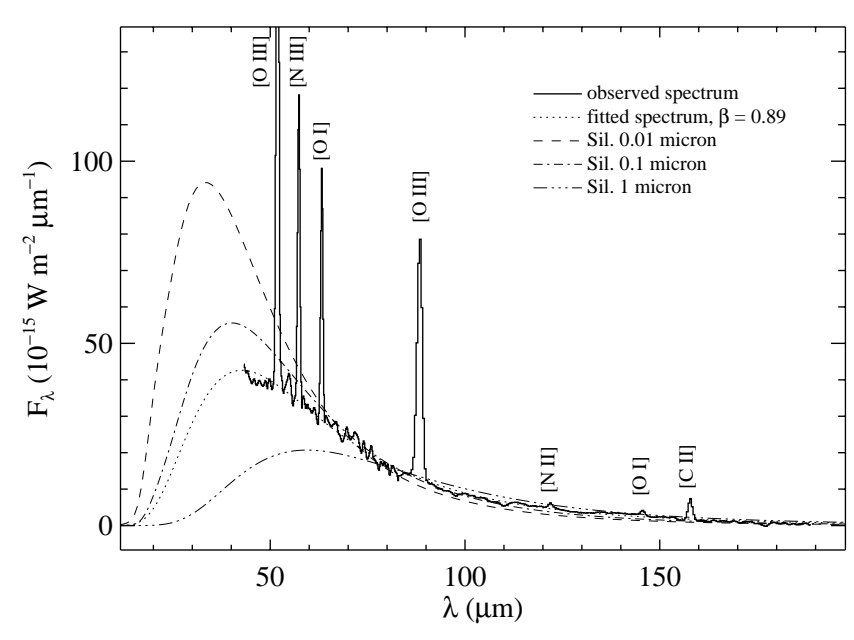

FIG. 7.-Far-infrared spectrum of NGC 6445

Waters et al. 1996), and a power-law approximation is not valid for such grains. In the on-source spectrum we do not detect any crystalline silicate features in the LWS range, but we cannot rule out the presence of such features below the detection limit in the SWS observations. In the absence of better data, we will assume that the power-law approximation is valid in the LWS range. This assumption is commonly made for fitting diffuse ISM emissions, often using $\beta=2$.

\subsubsection{The ISM Background}

Before we discuss the grain emission from the nebula itself, we discuss the fits to the ISM background emission. We derived estimates for the temperature of the warm and very cold components by using a two-component model. Each component has the same analytic dependence given above, and we assumed $\beta=2$ for both components. To constrain the fit we used the $60 \mu \mathrm{m}$ point from the HIRES image and the LWS off-source spectrum longward of 85 $\mu \mathrm{m}$. The optimal fit is shown in Figure 6 and Table 5. We find that the warm cirrus component has a temperature of $24 \mathrm{~K}$, which makes it possibly the warmest cirrus cloud known. Lagache et al. (1998) show various measurements in their Figure 2, the highest being $23 \mathrm{~K}$ in the Orion complex. The Galactic coordinates for our measurement are $l=8.119, b=+3.930$. Comparison with Figure 1 of Lagache et al. (1998) shows that they are in the middle of a high-temperature region. Since their data is smoothed over a $7^{\circ}$ beam, small clouds with higher temperatures could have been averaged out. Taken at face value, our detection of a very cold component of $7 \mathrm{~K}$ is in agreement the results of Wright et al. (1991) and Reach et al. (1995), who derived the widespread presence of such a component based on Far-Infrared Absolute Spectrophotometer (FIRAS) data taken by $C O B E$. Lagache et al. (1998) subsequently dismissed these claims upon a reinterpretation of DIRBE and FIRAS data. Since our observation encompasses only a small region of the sky, it is not clear how extended the $7 \mathrm{~K}$ component is and whether it could have contributed significantly to the FIRAS spectrum. Note also that our line of sight is in the Galactic plane. Given this fact, the very cold emission component may come from a starless core. WardThompson et al. (1994) have observed two such cores with temperatures of $8 \mathrm{~K}$. Bernard et al. (1992) predict that for a core to reach a temperature as low as $7 \mathrm{~K}$ the optical depth should be at least $A_{V}=100 \mathrm{mag}$. Therefore, for NGC 6445 to be observable, the core should reside behind the PN, giving a minimum distance of $1.5 \mathrm{kpc}$. Because we see the very cold component in both the on-source and off-source spectra, the angular extent must be at least $5^{\prime}$. This leads to a lower limit of $2 \mathrm{pc}$ for the diameter of the core. This is considerably larger than the cores observed by WardThompson et al. (1994). It is not clear whether cores this large can sustain a temperature of $7 \mathrm{~K}$ (see, e.g., the discussion in Laureijs 1999, and references therein). The ISOPHOT Serendipity Survey (ISOSS) also finds very cold cores with temperatures of approximately $13 \mathrm{~K}$ and a radial extent consistent with our observations (Hotzel et al. 1999). It should be noted that ISOSS can detect only cores with temperatures in the range $10 \mathrm{~K}<T_{\text {dust }}<70 \mathrm{~K}$ (Tóth et al. 1999). Hence the nondetection of cores with temperatures below $10 \mathrm{~K}$ in ISOSS is expected. Further observations are needed to clarify the nature of the very cold component.

\subsubsection{The Nebular Grains}

The LWS data for NGC 6445 show evidence for the presence of dust that is cooler than observed in most other planetary nebulae. We will investigate this emission first, before we try to determine where the emitting grains reside. The resulting best fit gave $\beta=0.89$ and is shown in Table 5 and Figure 7. Fits with $\beta$ fixed to other values are also shown for comparison in Table 5 . The total flux emitted by the grains, $F(\mathrm{IR})=2.38 \times 10^{-12} \mathrm{~W} \mathrm{~m}^{-2}$, is nearly a factor of two lower than was previously reported by Lenzuni, Natta, \& Panagia (1989) and Zhang \& Kwok (1993). This discrepancy can be attributed to the fact that both papers based their determination on the IRAS fluxes in all four bands. However, the IRAS 12 and $25 \mu \mathrm{m}$ detections of

TABLE 5

PARAMETERS FOR THE FITS TO THE FAR-INFRARED EMISSION (45-190 $\mu \mathrm{m})$

\begin{tabular}{|c|c|c|c|c|c|c|}
\hline \multirow[b]{2}{*}{$\beta$} & \multicolumn{3}{|c|}{ Nebular Grains } & \multicolumn{3}{|c|}{ ISM BACKGROUND } \\
\hline & $\begin{array}{l}T_{\text {dust }} \\
(\mathrm{K})\end{array}$ & $\begin{array}{c}\text { FIR } \\
\left(10^{-14} \mathrm{~W} \mathrm{~m}^{-2}\right)\end{array}$ & $\chi^{2} / N$ & $\begin{array}{l}T_{\text {warm }} \\
(\mathrm{K})\end{array}$ & $\begin{array}{l}T_{\text {cold }} \\
(\mathbf{K})\end{array}$ & $\chi^{2} / N$ \\
\hline $0.00 \ldots \ldots$ & 84.6 & 301 & 2.21 & $\cdots$ & $\ldots$ & $\cdots$ \\
\hline $0.50 \ldots \ldots$ & 66.5 & 259 & 1.59 & $\ldots$ & $\ldots$ & $\ldots$ \\
\hline $0.89 \ldots \ldots$ & 57.3 & 238 & 1.45 & $\ldots$ & $\ldots$ & $\ldots$ \\
\hline $1.00 \ldots \ldots$ & 55.2 & 233 & 1.46 & $\ldots$ & $\ldots$ & $\ldots$ \\
\hline $1.50 \ldots \ldots$ & 47.3 & 215 & 1.74 & $\ldots$ & $\ldots$ & $\ldots$ \\
\hline $2.00 \ldots \ldots$ & 41.5 & 201 & 2.32 & 24.4 & 6.7 & 0.92 \\
\hline
\end{tabular}


NGC 6445 are strongly dominated by line emission, which leads to an overestimation of the total infrared flux.

The temperature of $57 \mathrm{~K}$ for the nebular dust is considerably lower than what is typically observed in planetary nebulae. Dust grains with such typical temperatures (roughly between 90 and $150 \mathrm{~K}$ ) cannot be present in NGC 6445 , since they would be readily observable in the SWS spectrum. The ratio of the thermal grain emission to the $\mathrm{H} \beta$ flux of 27.0 is also very low. The presence of strong neutral lines in the spectrum indicates that the nebula is ionization bounded (see also $\S 5$ ). This argument is further strengthened by the fact that most determinations of the hydrogen and helium Zanstra temperatures for the central star are in good agreement (see Table 7). This is a good indication that the hydrogen Lyman continuum is optically thick. We therefore assume that no significant fraction of the Lyman continuum photons escape the nebula. Our photoionization model (see $\S 8)$ predicts $F(\operatorname{Ly} \alpha) / F(\mathrm{H} \beta)=26.6$, which implies that all of the grain heating could be accounted for by Ly $\alpha$ photons. Apparently Lyman continuum photons contribute little to grain heating and this suggests either that the ionized region is free of grains or that the ionization parameter is low, resulting in a low optical depth of the grains in the Lyman continuum (Bottorff et al. 1998). To investigate this further, we will first construct a photoionization model of the ionized region of NGC 6445.

\section{PHOTOIONIZATION MODELING}

We constructed a photoionization model of the ionized region using a modified version of CLOUDY 90.05 (Ferland et al. 1998) and following the method described in van Hoof \& Van de Steene (1999) with the following alterations. Instead of the blackbody assumption we used model atmospheres calculated by Rauch (1997). Furthermore, we used a constant hydrogen density throughout the ionized and neutral regions of the nebula. In view of the complex structure of the nebula, we did not constrain the model with an angular diameter but, alternatively, fixed the density at the value determined from plasma diagnostics. We also used the electron temperatures determined from various plasma diagnostics as additional constraints on the model. We assumed no dust grains to be present in the ionized region and did not constrain the model with infrared continuum fluxes. We omitted the infrared fine-structure lines of $\left[\mathrm{O}_{\mathrm{I}}\right]$ from the modeling input since preliminary models showed a large discrepancy between the modeled and observed fluxes of these lines. The observed fluxes are approximately a factor of 3-5 stronger than the model fluxes, and we feared that such a large discrepancy would upset the abundance determination. A possible explanation for this discrepancy could be provided by the fact that parts of the nebula are recombining, which would have a strong influence on the PDR physics. CLOUDY is not capable of modeling such nonequilibrium conditions, and an underestimation of the flux of the [ $\left[\begin{array}{ll}\mathrm{O} & \mathrm{I}\end{array}\right]$ fine-structure lines is expected because CLOUDY will underestimate the electron temperature in the recombining regions (also see the discussion in $\S 5$ ).

An alternative explanation could be the fact that the collision strengths for the [O I] fine-structure lines are poorly determined because they are difficult to calculate (Bhatia \& Kastner 1995). For instance, cross sections for $\mathrm{O}^{0}+e$ collisions have recently been revised downward by a factor of 5-50 (Bell, Berrington, \& Thomas 1998), based on calcu- lations valid only below $3000 \mathrm{~K}$. Cross sections for $\mathrm{O}^{0}+\mathrm{H}$ (Launay \& Roueff 1977) and $\mathrm{O}^{0}+\mathrm{He}$ (Monteiro \& Flower 1987) collisions are available only for temperatures below $1000 \mathrm{~K}$. Since the [O I] fine-structure lines are mainly formed in regions with electron temperatures between 4000 and $10,000 \mathrm{~K}$, significant extrapolations of these (possibly uncertain) data are needed. This makes modeling of the [O I] fine-structure lines very difficult and may very well explain the discrepancy between the observed and calculated values.

The parameters for the photoionization model we derived are listed in Table 6.

\subsection{The Central Star}

The temperature of the central star of NGC 6445 has been determined many times, mainly using the Zanstra method. An overview of all recently published determinations can be found in Table 7 . Most of these are based on a blackbody approximation. The average of all determinations using the Zanstra method, or a variation thereof, is $188 \pm 5 \mathrm{kK}$. This is in excellent agreement with the value that has been derived in this work, based on atmosphere models by Rauch (1997). This indicates that in the case of NGC 6445 the Zanstra method works well. This can be understood given the fact that the nebula is ionization bounded and optical depth effects due to dust grains are negligible. Hence the basic assumptions of the Zanstra method are valid in this case, and the main uncertainties in applying the method will stem from observational error and to a much lesser amount from assumptions concerning the shape of the central star spectrum (i.e., the blackbody assumption).

TABLE 6

PARAMETers For the ClOUDY Model of NGC 6445

\begin{tabular}{|c|c|c|}
\hline Parameter & Value & $\begin{array}{l}\text { Relative to } \\
\text { Solar }^{\mathrm{a}}\end{array}$ \\
\hline$T_{\text {eff }}(\mathrm{kK}) \ldots \ldots$ & 184. & \\
\hline$L_{*}\left(L_{\odot}\right) \ldots \ldots \ldots$ & 1035. & \\
\hline$r_{\text {in }}(\mathrm{mpc}) \ldots \ldots$ & 79. & \\
\hline$r_{\mathrm{Str}}(\mathrm{mpc}) \ldots \ldots$ & 183. & \\
\hline$n_{\mathrm{H}}\left(\mathrm{cm}^{-3}\right) \ldots \ldots$ & $525^{\mathrm{b}}$ & \\
\hline$T_{e}(\mathrm{kK}) \ldots \ldots \ldots$ & $11.49^{c}$ & \\
\hline$n_{e}\left(\mathrm{~cm}^{-3}\right) \ldots \ldots$ & $571^{\mathrm{c}}$ & \\
\hline $\boldsymbol{\epsilon}(\mathrm{He})^{\mathrm{d}} \ldots \ldots \ldots$ & 11.18 & +0.19 \\
\hline$\epsilon(\mathrm{C}) \ldots \ldots \ldots \ldots$ & 8.87: & +0.32 : \\
\hline$\epsilon(\mathrm{N}) \ldots \ldots \ldots \ldots$ & 8.39 & +0.42 \\
\hline$\epsilon(\mathrm{O}) \ldots \ldots \ldots \ldots$ & 8.87 & +0.00 \\
\hline$\epsilon(\mathrm{Ne}) \ldots \ldots \ldots \ldots$ & 8.31 & +0.23 \\
\hline$\epsilon(\mathrm{Mg}) \ldots \ldots \ldots . .$. & 7.23: & -0.35 : \\
\hline $\boldsymbol{\epsilon}(\mathrm{Si}) \ldots \ldots \ldots \ldots$ & 6.86 & -0.69 \\
\hline$\epsilon(\mathrm{S}) \ldots \ldots \ldots \ldots$ & 6.90 & -0.43 \\
\hline$\epsilon(\operatorname{Ar}) \ldots \ldots \ldots \ldots$ & 6.58 & +0.06 \\
\hline$\epsilon(\mathrm{Ca}) \ldots \ldots \ldots . .$. & $<5.09$ & $<-1.27$ \\
\hline$D(\mathrm{kpc}) \ldots \ldots \ldots$ & $1.5^{\mathrm{b}, \mathrm{e}}$ & \\
\hline$\chi^{2} \ldots \ldots \ldots \ldots$ & 2.9 & \\
\hline
\end{tabular}

NoTE.-All symbols have their usual meanings.

${ }^{a}$ Grevesse, Noels \& Sauval 1996.

b This parameter was not varied.

c This is a weighted average over the entire nebula.

${ }^{\mathrm{d}} \epsilon(A)$ stands for the logarithmic abundance of $A ; \epsilon(\mathrm{H}) \equiv 12$.

e Van de Steene \& Zijlstra 1995. 
TABLE 7

VARious Determinations of Stellar Temperature

\begin{tabular}{|c|c|c|c|}
\hline Source & $\begin{array}{c}T_{Z}(\mathrm{H} \mathrm{I}) \\
(\mathrm{kK})\end{array}$ & $\begin{array}{c}T_{Z}(\mathrm{He} \text { II }) \\
(\mathrm{kK})\end{array}$ & $\begin{array}{l}\text { Other Methods } \\
\qquad(\mathrm{kK})\end{array}$ \\
\hline $1 \ldots \ldots$ & $\ldots$ & 182. & $\ldots$ \\
\hline $2 \ldots \ldots$ & $\ldots$ & $\ldots$ & 174. (ZM) \\
\hline $3 \ldots \ldots$ & $\ldots$ & 184. & $\ldots$ \\
\hline $4 \ldots \ldots \ldots$ & 250. & 255. & $\ldots$ \\
\hline $5 \ldots \ldots$ & 184. & 186. & $\ldots$ \\
\hline $6 \ldots \ldots$ & $\ldots$ & $\ldots$ & 145. (EB) \\
\hline $7 \ldots \ldots$ & $\ldots$ & $\ldots$ & 180. (He) \\
\hline $8 \ldots \ldots$ & 175. & 175. & $\ldots$ \\
\hline $9 \ldots \ldots$ & 162. & 166. & $\ldots$ \\
\hline $10 \ldots \ldots$ & 224. & 186. & $\ldots$ \\
\hline $11 \ldots \ldots$ & $\ldots$ & $\ldots$ & 172. $(\mathrm{He})$ \\
\hline $12 \ldots \ldots$ & 183. & 215. & $\ldots$ \\
\hline $13 \ldots \ldots$ & $\ldots$ & $\ldots$ & 186. (ZA) \\
\hline $14 \ldots \ldots$ & $\ldots$ & $\ldots$ & 184. (ZC) \\
\hline $15 \ldots \ldots$ & 177. & 180. & $\ldots$ \\
\hline $16 \ldots \ldots$ & $\ldots$ & $\ldots$ & 194. (EB) \\
\hline $17 \ldots \ldots$ & 182. & 214. & 182. (ZC) \\
\hline $17 \ldots \ldots$ & 178. & 182. & $\ldots$ \\
\hline $18 \ldots \ldots$ & 174. & 159. & 166. (EB) \\
\hline
\end{tabular}

NotE.-Methods: EB: energy balance or Stoy method; He: empirical relation between $I(468.6 \mathrm{~nm})$ and $T_{*} ; \mathrm{ZA}$ : average of hydrogen and helium Zanstra temperature; ZC: Zanstra temperature using crossover technique; ZM: helium Zanstra temperature method using atmosphere models.

SouRCES. - (1) Harman \& Seaton 1966; (2) Pilyugin, Sakhibullin, \& Khromov 1978; (3) Köppen \& Tarafdar 1978; (4) Pottasch 1981; (5) Kaler 1983 (using a covering factor $\xi=1.00$ ); (6) Preite-Martinez \& Pottasch 1983; (7) Che \& Köppen 1983; (8) Reay et al. 1984; (9) Tylenda 1986; (10) Sabbadin 1986b; (11) Gurzadyan 1988; (12) Gathier \& Pottasch 1988; (13) Tylenda 1989; (14) Kaler \& Jacoby 1989; (15) Jacoby \& Kaler 1989; (16) Preite-Martinez et al. 1989; (17) Mallik 1991; (18) Mal'kov, Golovatyj, \& Rokach 1995.

In order to check our determination of the luminosity of the central star, we also made predictions for the magnitude of the central star in various photometric bands. A comparison of the results with observed magnitudes is shown in Table 8. The largest discrepancy is with the $V$ magnitude measured by Jacoby \& Kaler (1989). Their flux may be too low because of the possible presence of clouds during the observations. Gathier \& Pottasch (1988) used a narrowband filter that is free of emission lines. This technique is intrinsically more accurate than using a broadband filter like Johnson $V$. Since their measurement is in excellent agreement with the predictions, this confirms our determination of the central star luminosity with photoionization modeling.
When one compares the stellar temperature and luminosity with the theoretical calculations of Blöcker (1995a, $1995 \mathrm{~b}$ ), one finds that they indicate a core mass between 0.625 and $0.696 M_{\odot}$, or alternatively a main-sequence mass between 3 and $4 M_{\odot}$. The evolutionary ages of the nebula predicted by the Blöcker tracks are approximately 3500 and $900 \mathrm{yr}$ for the $0.625 M_{\odot}$ and $0.696 M_{\odot}$ tracks, respectively. This is in excellent agreement with the derived dynamical age of $3300 \mathrm{yr}$.

\subsection{Dust Grains in the Ionized Region}

Our model shows the carbon and oxygen abundances to be about equal. In view of the fact that the carbon abundance may be overestimated because of the uncertainties in the IUE data, it seems more likely that the nebula is oxygen rich. However, we take no final standpoint on this issue and leave both possibilities open. It is a well-known fact that in an oxygen-rich environment silicate grains can be formed. These grains predominantly contain oxides of magnesium, silicon, and iron, but also of other elements such as calcium, titanium, chromium, and nickel. It is a lesser known fact that these elements can also be depleted in a carbon-rich environment. See, for instance, Beintema et al. (1996), where it is shown that sodium, magnesium, aluminum, silicon, calcium, and iron are depleted in the carbon-rich nebula NGC 7027. It is unclear what types of grains these elements have been depleted into. They may either be carbides (e.g., $\mathrm{SiC}$ has been observed in various objects) or be locked up as impurities in more abundant amorphous carbon or graphite grains. Which of these grain types are present in NGC 6445 is unclear, so we will simply refer to them as siliconbearing grains.

First we will investigate whether grain destruction could have taken place in the ionized region of NGC 6445 . When dust grains are destroyed, the constituent material is returned to the gas phase, making the composition essentially solar again. This has a profound effect on the spectrum being emitted by the plasma. Many strong lines of elements like magnesium, aluminum, calcium, vanadium, and chromium should be easily detectable in UV or optical spectra (Kingdon, Ferland, \& Feibelman 1995; Kingdon \& Ferland 1997). The fact that none of these lines are detected in the spectrum of NGC 6445 already indicates that no substantial amount of grain destruction (at least for the silicon-bearing grains) can have taken place in the ionized region. This is confirmed by our photoionization modeling, which clearly indicates that elements like magnesium, silicon, and calcium are depleted in the ionized region. The derived abundance for magnesium is marked uncertain because it is based on only one emission line belonging to a subordinate ionization stage, which implies that a substan-

TABLE 8

Predicted and Observed Central Star Magnitudes

\begin{tabular}{cccccrr}
\hline \hline Data Set & $U$ & $B$ & $479.3 \mathrm{~nm}^{\mathrm{a}}$ & $V$ & $R$ & $I$ \\
\hline Model ......... & 14.82 & 16.30 & 16.43 & 16.66 & 16.85 & 17.10 \\
$A(\lambda)(\mathrm{mag}) \ldots \ldots$ & 3.24 & 2.74 & 2.52 & 2.14 & 1.68 & 1.14 \\
Predicted ....... & 18.06 & 19.04 & 18.95 & 18.80 & 18.53 & 18.24 \\
Observed ....... & $\ldots$ & $\ldots$ & $18.97^{\mathrm{b}}$ & $18.9,^{\mathrm{c}} 19.04^{\mathrm{d}}$ & $\ldots$ & $\ldots$ \\
\hline
\end{tabular}

${ }^{\text {a }}$ ESO 1402 filter centered on $479.3 \mathrm{~nm}$.

b Gathier \& Pottasch 1988.

c Reay et al. 1984.

d Jacoby \& Kaler 1989. 
tial correction is needed for unobserved ionization stages. By itself the magnesium underabundance is not convincing evidence for depletion. It is, however, supported by the underabundance for silicon, which is better determined. It is also based on only one emission line, [Si II] $34.8 \mu \mathrm{m}$, but since $\mathrm{Si}^{+}$is the dominant ionization stage, only a small correction for unobserved ionization stages is needed. Hence the dominant source of error for the abundance will be the uncertainty in the measured line flux and to a lesser extent the aperture correction. It is very unlikely that the strength of the silicon line could have been underestimated by a factor of 5 and it is even less likely that the aperture correction could be off by such a large factor. The calcium underabundance is based on a $3 \sigma$ upper limit for the [Ca II] $729.1 \mathrm{~nm}$ line. Since $\mathrm{Ca}^{+}$is a subordinate ionization stage, the largest source of error will be the correction for unobserved ionization stages. It seems highly unlikely however that this correction could be off by a factor of 20 .

Recently Liu (1998) published an abundance analysis of the planetary nebula NGC 6153 , which yielded abundances derived from recombination lines that were roughly a factor of 10 higher than those derived from collisionally excited forbidden lines. Taken at face value, this could cast doubt on the analysis presented above. Similar discrepancies have been observed in other nebulae as well, but usually they are much less pronounced. The strength of this effect for NGC 6445 has not been established. Whether such a discrepancy exists for magnesium, silicon, or calcium has also not been established since the recombination lines of these lines are too weak to be observable. The physics causing the discrepancy between collisional and recombination abundances is not clear, and therefore both values should be viewed with caution until such an explanation is at hand. Liu (1998) claims that there is no evidence that the recombination abundances have been grossly overestimated. However, the derived $\mathrm{O}^{2+}$ abundance of 9.65 for NGC 6153 is much larger than the solar oxygen abundance. Significant enhancement of oxygen in post-AGB stars is not expected by stellar evolution theory. We argue that this fact does cast doubt on the recombination abundances. On the other hand, the $\mathrm{O}^{2+}$ abundance derived by Liu (1998) from forbidden lines (using essentially the same method we use in this paper) is in good agreement with the expected solar value. Exactly the same argument holds for the $\mathrm{Ne}^{2+}$ abundances of NGC 6153. In view of these facts it seems fair to assume that the collisional abundances derived in this work are valid. Therefore we claim that there is evidence for depletion in NGC 6445, which in turn proves that the dust grains that contain these elements cannot have been destroyed in situ, at least not in significant amounts.

An alternative assumption could be that the ionized region is free of dust grains because of the fact that the grains were separated from the gas. If dust-gas separation occurred, it must have been during the post-AGB phase. It could not have occurred during an earlier phase, since then dust formation would still be ongoing. On the other hand, separation during the early post-AGB phase could be expected as it is radiation pressure on dust grains that is thought to drive the outflow of gas and dust at that stage. However, this phase is very short lived, and it is not likely that a detectable amount of dust-gas separation could occur in that period. Separation during later phases seems impossible since our photoionization model indicates that once the nebular material is ionized the drift velocity with respect to the gas is lower than $1 \mathrm{~km} \mathrm{~s}^{-1}$. This velocity is far too low to produce any significant dust-gas separation.

We have constructed a model for the grain emission of NGC 6445 based on the assumption that it contains grains at a constant dust-to-gas ratio throughout the nebula. Heating of the grains by Ly $\alpha$ photons must be included in this model. Ly $\alpha$ photons are scattered very efficiently by neutral hydrogen because of Rayleigh scattering. This process tends to lock up the photons inside the neutral envelope of the nebula until they are eventually absorbed by grain particles. However, Ly $\alpha$ photons can escape provided they can pick up sufficient redshift by repeatedly being bounced off the expanding neutral envelope. We have included a simplified analytic treatment of this process in the model. This treatment assumes that there is a sharp boundary between a fully ionized and a fully neutral region. Hence there is no Rayleigh scattering inside the ionized region, only outside. It is known that this is not a good approximation, and it will overestimate the escape probability to some extent. We also included sources of grain heating other than $L y \alpha$ in our treatment by using the CLOUDY model. These calculations indicate that roughly $25 \%$ of the total grain heating is due to sources other than Ly $\alpha$ and hence that roughly $25 \%$ of the Ly $\alpha$ photons must escape in order to account for the observed total grain emission. This shows that Ly $\alpha$ escape should be included in the model. Given that there are considerable uncertainties in the shape of the absorption law for the grains and in the geometry of the nebula, we felt that a more detailed treatment of Ly $\alpha$ scattering (e.g., using a Monte Carlo code) was not warranted. The shape of the absorption law is important because the cross section at $121.6 \mathrm{~nm}$ (combined with the dust-to-gas ratio) determines the escape probability and because a large infrared emissivity (when compared to the UV absorption) will tend to make the grains cooler. Also deviations from $\beta=2$ in the LWS range will have an important effect on the modeling. None of these parameters are accurately known for the grains in NGC 6445.

We produced three models using single-sized grains of astronomical silicate (Martin \& Rouleau 1991) with diameters $0.01,0.1$, and $1 \mu \mathrm{m}$. We treated the dust-to-gas ratio as a free parameter but assumed it to be constant as a function of radius. The results are shown in Figure 7. The dust-to-gas ratios for the best-fitting models are $3.0 \times 10^{-3}(0.01 \mu \mathrm{m})$, $4.1 \times 10^{-3}(0.1 \mu \mathrm{m})$, and $1.7 \times 10^{-2}(1 \mu \mathrm{m})$. The models using the 0.01 and $1 \mu \mathrm{m}$ grains are clearly unrealistic, but the $0.1 \mu \mathrm{m}$ grains give a reasonable fit. The sequence of models suggests that the grains in NGC 6445 are on average large.

We mentioned earlier that our model will overestimate the escape probability of $\mathrm{Ly} \alpha$ photons to some extent. Since the total amount of heating of the grains is constrained by the observations, the model fitted to the observations will tend to overestimate the dust-to-gas ratio somewhat to counteract this effect. If we had assumed the silicon-bearing grains to be crystalline silicates instead of astronomical silicate and if we had assumed the UV absorption characteristics of both silicate types to be the same, the crystalline silicates would have been cooler because of the presence of strong emission features in the mid- and far-infrared. Under those conditions our model sequence would have led to a somewhat lower estimate for the average grain size and the dust-to-gas ratio. Using graphite grains would have led to a model and a dust-to-gas ratio that are roughly similar to 
those quoted above. Given the uncertainties in our model and the absorption and emission characteristics of the grains in NGC 6445, we cannot derive precise results. However, it is very likely that the grains in NGC 6445 are large and that the dust-to-gas ratio is several times $10^{-3}$. One can also predict the dust-to-gas ratio assuming that the silicon abundance before grain formation was solar and that the grains are composed of pure $\mathrm{MgSiFeO}_{4}$. This yields $3.2 \times 10^{-3}$, in excellent agreement with our results. We find there is no need to invoke dust-gas separation in this nebula in order to obtain a good fit to the spectrum.

Therefore, the most likely conclusion is that grains are residing inside the ionized region of NGC 6445 and that the low temperature is caused by the low luminosity of the central star combined with the large extent of the nebula. The low flux of the grain emission, when compared to $\mathrm{H} \beta$, is caused by the low optical depth of the grains in the Lyman continuum, which makes Ly $\alpha$ photons the dominant heating source for the grains. In short, all of these characteristics are a consequence of the fact that the nebula is well evolved. Our conclusion implies that the bulk of the siliconbearing grains in this nebula could survive exposure to hard-UV photons for at least several thousands of years. This contradicts the results of Natta \& Panagia (1981) and Pottasch (1987), who find a tight correlation between the dust-to-gas ratio and the nebular radius based on a small sample of PNe. The dust-to-gas ratio in NGC 6445 is much higher than would be expected from this correlation. The results given by Natta \& Panagia (1981) predict a dust-togas ratio of approximately $2 \times 10^{-5}$ for NGC 6445 , i.e., roughly 2 orders of magnitude lower than what we find. We are confident that our models could not have overestimated the dust content of the nebula by such a large factor. We cannot rule out that some destruction of the smallest grains, constituting only a small mass fraction, could have occurred. Small grains would be the first to be destroyed since they absorb UV radiation more effectively and are therefore hotter than larger grains. Such an occurrence would cause the grains in NGC 6445 to be on average larger. It is not clear whether this is sufficient to explain the large average grain size discussed above or whether it is simply the natural result of grain growth (and coagulation) during the prior AGB phase. Natta \& Panagia (1981) found a tight decreasing relation between the average grain size and the nebular radius based on the same sample mentioned earlier. This relation would predict an average grain size of $0.007 \mu \mathrm{m}$ for NGC 6445 , which is significantly smaller than what we find. Hence this prediction also seems implausible.

\section{CONCLUSIONS}

1. We presented ISO-SWS and LWS spectra of the planetary nebula NGC 6445 that indicate the presence of very cool dust with a low flux compared to $\mathrm{H} \beta$. The fact that $\mathrm{Ly} \alpha$ photons alone have sufficient energy to account for all of the grain heating suggests that either the optical depth of the grains in the Lyman continuum is very low or that the ionized region is free of grains.

2. A photoionization model of the ionized region of NGC 6445 was constructed. Based on this model we showed that it is very unlikely that either grain destruction or dust-gas separation has taken place in the ionized region.

3 . Hence, the most likely conclusion is that grains reside inside the ionized region of NGC 6445 and that the low temperature and flux of the grain emission is caused by the low luminosity of the central star and the low optical depth of the grains. This implies that the bulk of the siliconbearing grains in this nebula could survive exposure to hard-UV photons for several thousands of years, contradicting previously published results.

4. A comparison between optical and infrared diagnostic line ratios give a marginal indication for the presence of a $t^{2}$ effect. However, the evidence is not convincing and alternative explanations could be provided by uncertainties in the absolute flux calibration of the spectra, the aperture corrections that have been applied, or the collisional cross sections.

5. The photoionization model allows an accurate determination of the central star temperature using model atmospheres by Rauch (1997). The resulting value of $184 \mathrm{kK}$ is in good agreement with the average of all published Zanstra temperatures.

6. The off-source spectrum taken with LWS clearly shows the presence of a warm cirrus component with a temperature of $24 \mathrm{~K}$ as well as a very cold component with a temperature of $7 \mathrm{~K}$. Since our observation encompasses only a small region of the sky, it is not clear how extended the $7 \mathrm{~K}$ component is and whether it contributed significantly to the FIRAS spectrum taken by $C O B E$, as reported by Wright et al. (1991) and Reach et al. (1995) but dismissed by Lagache et al. (1998). Because our line of sight is in the Galactic plane, the very cold component could be a starless core.

This research was partially based on observations obtained at the European Southern Observatory and on observations with $I S O$, an ESA project with instruments funded by ESA Member States (especially the PI countries: France, Germany, the Netherlands, and the United Kingdom) and with the participation of ISAS and NASA. We thank the NSF and NASA for support through grants AST 96-17083 and GSFC-123. We thank Dr. A. Lazarian for stimulating discussions and C. Kerton for processing the IRAS data. The Canadian Institute for Theoretical Astrophysics is thanked for hospitality and financial support during our stay in Toronto.

\section{REFERENCES}

Aller, L. H., Czyzak, S. J., Craine, E., \& Kaler, J. B. 1973, ApJ, 182, 509 Beintema, D. A., et al. 1996, A\&A, 315, L253

Bell, K. L., Berrington, K. A., \& Thomas, M. R. J. 1998, MNRAS, 293, L83 Bernard, J. P., Boulanger, F., Desert, F. X., \& Puget, J. L. 1992, A\&A, 263, 258

Bhatia, A. K., \& Kastner, S. O. 1995, ApJS, 96, 325

Blöcker, T. 1995a, A\&A, 297, 727

.1995b, A\&A, 299, 755

Bottorff, M., LaMothe, J., Momjian, E., Verner, E., Vinković, D., \&

Ferland, G. 1998, PASP, 110, 1040
Butler, K., \& Zeippen, C. J. 1994, A\&AS, 108, 1

Cardelli, J. A., Clayton, C., \& Mathis, J. S. 1989, ApJ, 345, 245

Che, A., \& Köppen, J. 1983, A\&A, 118, 107

Clegg, P. E., et al. 1996, A\&A, 315, L38

Corradi, R. L. M., \& Schwarz, H. E. 1995, A\&A, 293, 871

de Graauw, Th., et al. 1996, A\&A, 315, L49

Dinerstein, H. L., Lester, D. F., \& Werner, M. W. 1985, ApJ, 291, 561

Draine, B. T., \& Lee, H. M. 1984, ApJ, 285, 89

Ferland, G. J., Korista, K. T., Verner, D. A., Ferguson, J. W., Kingdon,

J. B., \& Verner, E. M. 1998, PASP, 110, 761 
Galavis, M. E., Mendoza, C., \& Zeippen, C. J. 1995, A\&AS, 111, 347 1997, A\&AS, 123, 159

Gathier, R., \& Pottasch, S. R. 1988, A\&A, 197, 266

Grevesse, N., Noels, A., \& Sauval, A. J. 1996, in ASP Conf. Ser. 99, Cosmic

Abundances, ed. S. S. Holt \& G. Sonneborn (San Francisco: ASP), 117

Gurzadyan, G. A. 1988, Ap\&SS, 149, 343

Harman, R. J., \& Seaton, M. J. 1966, MNRAS, 132, 15

Hotzel, S., et al. 1999, in The Universe as Seen by ISO, ed. P. Cox \& M. F. Kessler (ESA SP-427; Noordwijk: ESA), 675

Huggins, P. J., \& Healy, A. P. 1989, ApJ, 346, 201

Jacoby, G. H., \& Kaler J. B. 1989, AJ, 98, 1662

Kaler, J. B. 1983, ApJ, 271, 188

Kaler, J. B., \& Jacoby, G. H. 1989, ApJ, 345, 871

Kingdon, J., Ferland, G. J., \& Feibelman, W. A. 1995, ApJ, 439, 793

Kingdon, J. B., \& Ferland, G. J. 1997, ApJ, 477, 732

Köppen, J., \& Tarafdar, S. P. 1978, A\&A, 69, 363

Lagache, G., Abergel, A., Boulanger, F., \& Puget, J.-L. 1998, A\&A, 333, 709

Launay, J. M., \& Roueff, E. 1977, A\&A, 56, 289

Laureijs, R. J. 1999, in The Universe as Seen by ISO, ed. P. Cox \& M. F. Kessler (ESA SP-427; Noordwijk: ESA), 599

Lennon, D. J., \& Burke, V. M. 1994, A\&AS, 103, 273

Lenzuni, P., Natta, A., \& Panagia, N. 1989, ApJ, 345, 306

Liu, X.-W. 1998, Ap\&SS, 255, 499

Mal'kov, Y. F., Golovatyj, V. V., \& Rokach, O. V. 1995, Ap\&SS, 232, 99

Mallik, D. C. V. 1991, Proc. Astron. Soc. Australia, 9, 15

Martin, P. G., \& Rouleau, F. 1991, in Extreme Ultraviolet Astronomy, ed.

R .F. Malina \& S. Bowyer (New York: Pergamon Press), 341

Mathis, J. S. 1990, ARA\&A, 28, 37

Mendoza, C., \& Zeippen, C. J. 1982, MNRAS, 199, 1025

Milne, D. K., \& Aller, L. H. 1982, A\&AS, 50, 209

Monteiro, T. S., \& Flower, D. R. 1987, MNRAS, 228, 101

Natta, A., \& Panagia, N. 1981, ApJ, 248, 189

O'Dell, C. R. 1963, ApJ, 138, 293

Oliva, E., Pasquali, A., \& Reconditi, M. 1996, A\&A, 305, L21

Peimbert, M. 1967, ApJ, 150, 825

Perinotto, M. 1991, ApJS, 76, 687

Phillips, J. P. \& Mampaso, A. 1988, A\&A, 190, 237

Pilyugin, L. S., Sakhibullin, N. A., \& Khromov, G. S. 1978, Astrophysics, 14,377

Pottasch, S. R. 1981, A\&A, 94, L13

1984, Planetary Nebulae (Dordrecht: Reidel)
Pottasch, S. R. 1987, in Late Stages of Stellar Evolution, ed S. Kwok \& S. R. Pottasch (Dordrecht: Reidel), 355

Preite-Martinez, A., Acker, A., Köppen, J., \& Stenholm, B. 1989, A\&AS, 81,309

Preite-Martinez, A., \& Pottasch, S. R. 1983, A\&A, 126, 31

Rauch, T. 1997, A\&A, 320, 237

Reach, W. T., et al. 1995, ApJ, 451, 188

Reay, N. K., Pottasch, S. R., Atherton, P. D., \& Taylor, K. 1984, A\&A, 137, 113

Rieke, G. H., \& Lebofsky, M. J. 1985, ApJ, 288, 618

Rowlands, N., Houck, J. R., Skrutskie, M. F., \& Shure, M. 1993, PASP, 105,1287

Sabbadin, F. 1986a, A\&AS, 64, 579 1986b, A\&A, 160, 31

Schwarz, H. E., Corradi, R. L. M., \& Melnick, J. 1992, A\&AS, 96, 23

Shaw, R. A., \& Dufour, R. J. 1995, PASP, 107, 896

Simpson, J. P. 1975, A\&A, 39, 43

Storey, P. J., \& Hummer, D. G. 1995, MNRAS, 272, 41

Tóth, L. V., et al. 1999, in The Universe as Seen by ISO, ed. P. Cox \& M. F. Kessler (ESA SP-427; Noordwijk: ESA), 771

Tylenda, R. 1986, A\&A, 156, 217

. 1989, in IAU Symp. 131, Planetary Nebulae, ed. S. TorresPeimbert (Dordrecht: Kluwer), 531

Van de Steene, G. C., \& Zijlstra, A. A. 1995, A\&A, 293, 541

van Hoof, P. A. M., Beintema, D. A., Verner, D. A., \& Ferland, G. J. 2000, A\&A, in press

van Hoof, P. A. M., Oudmaijer, R. D., \& Waters L. B. F. M. 1997a, MNRAS, 289, 371

van Hoof, P. A. M., \& Van de Steene, G. C. 1999, MNRAS, 308, 627

van Hoof, P. A. M., Verner, D. A., Beintema, D. A., \& Ferland, G. J. 1997b, in Proc. First ISO Workshop on Analytical Spectroscopy, ed. A. M. Heras, K. Leech, N. R. Trams, \& M. Perry (ESA SP-419; Noordwijk: ESA), 235

Ward-Thompson, D., Scott, P. F., Hills, R. E., \& André, P. 1994, MNRAS, 268, 276

Waters, L. B. F. M., et al. 1996, A\&A, 315, L361

Watson, D. M., \& Storey, J. W. V. 1980, Int. J. Infrared Millimeter Waves, 1,60

Weinberger, R. 1989, A\&AS, 78, 301

Wright, E. L., et al. 1991, ApJ, 381, 200

Zhang, C. Y., \& Kwok, S. 1993, ApJS, 88, 137 